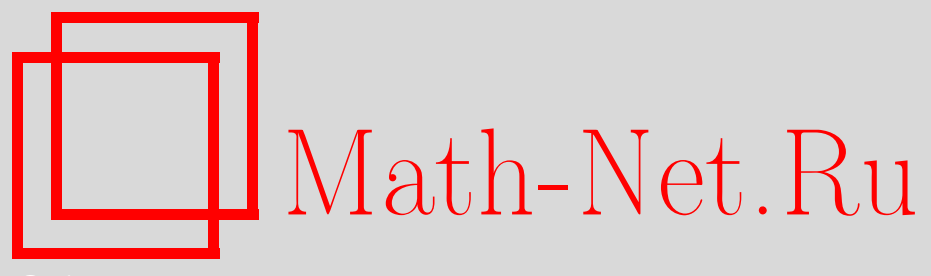

В. В. Козлов, Обобщенное кинетическое уравнение Власова, УМН, 2008, том 63, выпуск 4, 93-130

DOI: https://doi.org/10.4213/rm9216

Использование Общероссийского математического портала Math-Net.Ru подразумевает, что вы прочитали и согласны с пользовательским соглашением http://www . mathnet.ru/rus/agreement

Параметры загрузки:

IP: 54.209 .52 .79

26 апреля 2023 г., 04:43:21

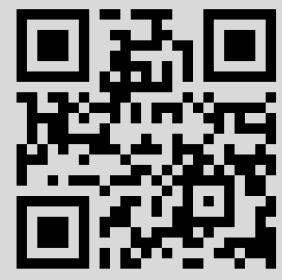




\title{
Обобщенное кинетическое уравнение Власова
}

\author{
В. В. Козлов
}

Работа посвящена изучению обобщенного кинетического уравнения, описывающего эволюцию плотности вероятностной меры. В общем случае оно является нелинейным интегро-дифференциальным уравнением. С одной стороны, это уравнение включает как частный случай более простое линейное уравнение Лиувилля (которое является основой классической статистической механики) и уравнение самосогласованного поля (кинетическое уравнение Власова), а с другой - к нему сводятся некоторые другие известные уравнения, в частности уравнение вихря для плоских течений идеальной несжимаемой жидкости. Основная цель работы - исследование задачи о слабых пределах решений обобщенного кинетического уравнения при неограниченном возрастании времени. Эта задача имеет существенное значение при переходе от микро- к макроописанию, когда изучается поведение средних (наиболее вероятных) значений динамических величин. Теория слабых пределов решений уравнения Лиувилля тесно связана с идеями и методами эргодической теории. Рассматриваемый случай представляет бо́льшие трудности, упирающиеся в нетривиальную проблему существования инвариантных счетно-аддитивных мер динамических систем в бесконечномерных пространствах. Результаты общего характера применяются к изучению континуумов взаимодействующих частиц и статистических свойств плоских течений идеальной жидкости.

Библиография: 42 названия.

\section{СОДЕРЖАНИЕ}

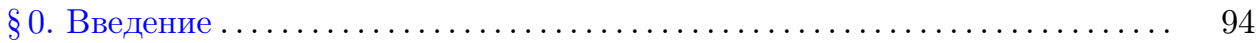

$\S 1$. Кинетическое уравнение Власова и его обобщения ............... 96

$\S 2$. Динамика континуума осцилляторов ...................... 99

$\S 3$. Уравнение вихря 2D-гидродинамики как кинетическое уравнение ... 101

$\S 4$. Классические и обобщенные решения .................... 102

$\S 5$. Кинетическое уравнение как динамическая система................ 105

$\S 6$. Слабые пределы решений обобщенного кинетического уравнения.... 108

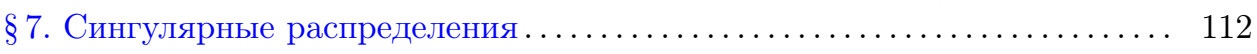

Работа выполнена при поддержке NWO-RFBR (грант № 047.011.2004.059) и программы "Ведущие научные школы" (грант № НШ-691.2008.1).

(C) В. В. Козлов, 2008 
$\S 8$. Неравенства для решений кинетического уравнения ............... 117

$\S 9$. Грубая энстрофия................................ 120

$\S 10$. Тождество Лагранжа ................................ 124

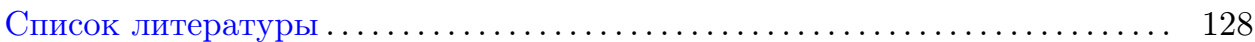

\section{§ 0. Введение}

Кинетические уравнения приближенно описывают эволюцию функции распределения частиц $\rho_{t}$ по координатам и скоростям. Простейшее из них - это классическое уравнение Лиувилля, которое определяет динамику континуума невзаимодействующих частиц, движущихся в заданном силовом поле (ансамбль Гиббса). Несмотря на свою простоту, уравнение Лиувилля лежит в основе всей классической статистической механики. С другой стороны, кинетика бесстолкновительной сплошной среды в замкнутых областях - весьма интересный и нетривиальный объект. Например, согласно Пуанкаре, независимо от начальной плотности распределения (лишь бы она была суммируемой функцией) бесстолкновительный газ в прямоугольном ящике с зеркальными стенками необратимо стремится равномерно заполнить этот ящик (как при $t \rightarrow+\infty$, так и при $t \rightarrow-\infty)$.

В зависимости от упрощающих предположений кинетические уравнения имеют различную форму. В приближении малой плотности кинетику упруго сталкивающихся маленьких шариков принято описывать классическим уравнением Больцмана. Хотя уравнение Больцмана приближенное (в частности, необратимое), оно качественно правильно описывает кинетику на определенных характерных временны́х интервалах.

В приближении среднего поля выводится кинетическое уравнение Власова, а в приближении слабого взаимодействия - уравнение Ландау (см., например, [1]).

Уравнение Власова и его естественные обобщения - предмет рассмотрения настоящей работы. Оно обладает одним важным и естественным свойством: среди его обобщенных решений есть взвешенные суммы $\delta$-функций Дирака. При этом сингулярности интерпретируются как частицы, коэффициенты при $\delta$-функциях - как массы этих частиц. Оказывается, динамика сингулярностей в точности определяется решениями уравнений Ньютона, описывающих взаимодействие конечного числа частиц. Таким образом, теория уравнения Власова - это далеко идущее и естественное расширение обычной классической механики.

С другой стороны, уравнения типа Власова появляются в разных иных ситуациях. В частности, уравнение вихря плоской гидродинамики идеальной несжимаемой жидкости приводится к обобщенному кинетическому уравнению Власова.

Одна из ключевых задач кинетики и статистической механики - понять механизм стремления изолированной системы к состоянию статистического равновесия. В этой задаче имеется существенная трудность: плотность $\rho_{t}$ как функция времени, как правило, осциллирует и вообще не стремится к чему-либо в обычном смысле. Выход состоит в замене обычной сходимости более 
сильным методом суммирования. Например, в эргодической теории обычно используют сходимость по Чезаро (средних арифметических). С другой стороны, при переходе от микро- к макроописанию существенную роль играет анализ поведения средних (наиболее вероятных) значений динамических величин. Таким образом, мы приходим к идее заменить обычную сходимость слабой сходимостью:

$$
\int_{M} \rho_{t} \varphi d \nu \rightarrow \int_{M} \bar{\rho} \varphi d \nu
$$

для любой “пробной” функции $\varphi$ на фазовом пространстве $M$ с "мерой Лиувилля" $d \nu$; функции $\varphi$ - это динамические величины, а $\bar{\rho}-$ слабый предел плотности $\rho_{t}$ при $t \rightarrow \infty$. Чтобы охватить бо́льшую часть содержательных примеров, сходимость по времени в (0.1) следует понимать по Чезаро.

Теория слабой сходимости решений уравнения Лиувилля для нелинейных гамильтоновых систем изложена в книгах [2], [3] (там можно найти дальнейшие ссылки). Она связана с модификацией некоторых классических идей эргодической теории [4], [5] и позволяет, в частности, развить последовательную теорию стремления изолированных систем к состоянию статистического равновесия (в рамках подхода Гиббса).

Задача о слабой сходимости решений обобщенного кинетического уравнения представляет бо́льшие трудности. Наш подход состоит в следующем. Кинетическое уравнение относительно плотности $\rho$ рассматривается как динамическая система в подходящем бесконечномерном функциональном пространстве (например, в $C^{k}(M), k \geqslant 0$, или $L_{p}(M, d \nu), p \geqslant 1$ ). Далее рассматриваем функционал

$$
\rho \mapsto \int_{M} \rho \varphi d \nu
$$

определяемый “пробной” функцией $\varphi$, и пытаемся воспользоваться индивидуальной эргодической теоремой Биркгофа-Хинчина. Эта идея упирается в нетривиальную и содержательную проблему наличия у рассматриваемой динамической системы "хорошей” инвариантной счетно-аддитивной меры. К сожалению, задача об инвариантных мерах пока решена лишь при некоторых специальных ограничениях. Кстати сказать, известные общие результаты (типа теоремы Крылова-Боголюбова) здесь не применимы ввиду некомпактности бесконечномерных пространств.

Правда, с физической точки зрения здесь нет особых проблем, поскольку любую меру на компактном фазовом пространстве $M$ можно сколь угодно точно приблизить (в слабом смысле) сингулярной мерой. В этом случае динамика сингулярностей описывается конечномерной динамической системой с инвариантной мерой, и мы можем воспользоваться стандартными методами эргодической теории.

Результаты общего характера иллюстрируются на двух содержательных примерах: кинетическое уравнение Власова и статистическая теория плоских течений идеальной несжимаемой жидкости.

Основная цель обзора - привлечь читателей к кругу задач, связанных со слабой сходимостью решений нелинейных интегро-дифференциальных уравнений, 
которые имеют важное значение для статистической механики и ее приложений. Ряд гипотез и нерешенных задач сформулированы в тексте статьи.

\section{§ 1. Кинетическое уравнение Власова и его обобщения}

Пусть $M$ - гладкое $n$-мерное многообразие с локальными координатами $\left(x_{1}, \ldots, x_{n}\right)=x, \rho_{t}(x)=\rho(x, t)-$ плотность меры $\mu\left(d \mu=\rho d^{n} x, d^{n} x=\right.$ $\left.d x_{1} \cdots d x_{n}\right)$, которая удовлетворяет следующему дифференциально-интегральному уравнению

$$
\frac{\partial \rho}{\partial t}+\sum_{i=1}^{n} \frac{\partial\left(\rho v_{i}\right)}{\partial x_{i}}=0, \quad v_{i}=\int_{M} K_{i}(x, y) \rho(y, t) d^{n} y .
$$

Компоненты векторного поля $\left(v_{1}, \ldots, v_{n}\right)=v$ - интегральные функционалы от плотности $\rho$ с ядрами $K_{i}: M \times M \rightarrow \mathbb{R}$. Как правило, в дальнейшем предполагается, что $K_{i}$ - гладкие функции, хотя в приложениях часто встречаются случаи, когда ядра имеют сингулярности на диагонали $\{x=y\} \subset M \times M$. Обычно (но не всегда) предполагается, что $K(x, x)=0$ для всех $x \in M$. В физических приложениях это означает отсутствие “самодействия".

Всюду ниже предполагается, что

$$
\sum_{i=1}^{n} \frac{\partial K_{i}}{\partial x_{i}}=0
$$

для всех $x, y$. Это - существенное условие. В частности, поток системы обыкновенных дифференциальных уравнений

$$
\dot{x}_{j}=v_{j}(x, t), \quad 1 \leqslant j \leqslant n,
$$

где компоненты поля $v$ определяются уравнением (1.1), сохраняет "стандартную" меру $d \nu=d^{n} x$.

Уравнение (1.1) является “уравнением неразрывности” для системы (1.3). Следовательно, ее поток также сохраняет меру $\mu$. В частности,

$$
\int_{M} \rho_{t}(x) d^{n} x=\text { const. }
$$

Это обстоятельство позволяет нам ограничиться рассмотрением вероятностных мер: интеграл (1.4) равен единице при всех $t$. В предположении (1.2) плотность $\rho=d \mu / d \nu$ - первый интеграл системы (1.3) (поскольку $\operatorname{div} v=0$ ). Поэтому первое уравнение (1.1) можно записать в виде

$$
\frac{\partial \rho}{\partial t}+\sum \frac{\partial \rho}{\partial x_{i}} v_{i}=0
$$

Если ядра $K_{i}$ не зависят от второй переменной, то система (1.3) становится автономной: $v_{j}=K_{j}(x)$. В этом случае уравнение (1.1) превращается в классическое уравнение Лиувилля, которое управляет эволюцией ансамбля Гиббca [6]. В частности, в рамках теории ансамблей Гиббса описывается динамика 
континуумов невзаимодействующих частиц. Уравнение Лиувилля - исходный пункт классической статистической механики (в том числе - теории цепочек уравнений Боголюбова) (см., например, [7]). При таком подходе вероятность вводится только посредством задания плотности распределения в начальный момент времени.

С другой стороны, уравнение (1.1) обобщает хорошо известное в статистической механике кинетическое уравнение Власова, которое также называется уравнением самосогласованного поля [8]. Это уравнение описывает эволюцию функции распределения $\rho(x, v, t)$ континуума взаимодействующих частиц в евклидовом пространстве по скорости $v$ и координате $x$ в момент времени $t$. Оно имеет вид

$$
\begin{gathered}
\frac{\partial \rho}{\partial t}+\left(\frac{\partial \rho}{\partial x}, v\right)+\left(\frac{\partial \rho}{\partial v}, F\right)=0 \\
F=-\frac{\partial}{\partial x} \int K(x, y) \rho(y, u, t) d u d y
\end{gathered}
$$

Здесь $K$ - парный потенциал взаимодействия, который в реальных задачах зависит от расстояния $|x-y|$, а $F$ - суммарная сила, с которой все частицы действуют на одну из них, находящуюся в момент времени $t$ в точке $x$. Уравнение (1.1) (записанное в форме (1.5)) будет иметь вид уравнения Власова (1.6), если предположить, что половина ядер $\left\{K_{j}\right\}$ не зависит от переменной $y$.

Обсуждение круга вопросов, связанных с существованием и гладкостью решений уравнения (1.1), мы отложим до §4. А пока не будем обращать на это внимания и обсудим простые (но важные) свойства обобщенного кинетического уравнения Власова.

Зафиксируем одно из "нормированных" решений $\rho_{t}$ уравнения (1.1). Оно порождает систему дифференциальных уравнений (1.3), в общем случае, конечно, неавтономную.

ТЕОРема 1.1. Если $f_{t}(x)$ - первый интеграл системы (1.3) и М компактно, mo

$$
J(t)=\int_{M} f_{t}(x) d^{n} x=\text { const. }
$$

Доказательство. Так как $\operatorname{div} v=0$, то функция $f$ удовлетворяет уравнению неразрывности

$$
\frac{\partial f}{\partial t}+\operatorname{div}(f v)=0
$$

Следовательно,

$$
\dot{J}=\frac{\partial}{\partial t} \int_{M} f_{t}(x) d^{n} x=-\int_{M} \operatorname{div}(f v) d^{n} x=0
$$

по теореме Гаусса-Остроградского ввиду замкнутости $M$.

ЗАмЕчАниЕ. В некомпактном случае заключение теоремы останется справедливым, если дополнительно потребовать равенства нулю потока поля $f v$ "на бесконечности".

СлЕДСтвиЕ 1.1. Интеграл (1.4) не меняется со временем. 
СлЕДСТвиЕ 1.2. Если $f$ - измеримая функиия одного переменного, то

$$
\int_{M} f\left(\rho_{t}(x)\right) d^{n} x=\text { const. }
$$

Чтобы это утверждение было содержательным, надо потребовать конечность интеграла (1.7).

СлЕДСтвиЕ 1.3. Энтропия

$$
-\int_{M} \rho_{t} \ln \rho_{t} d^{n} x
$$

не меняется со временем.

Поскольку для уравнения Власова (1.6) $\rho_{t}$ - это плотность “одночастичного" распределения (по терминологии физиков - распределение в $\mu$-пространстве), то интеграл (1.8) - аналог энтропии Больцмана. Однако в модели самосогласованного поля она, вопреки ожиданию, постоянна. Это - строгое следствие кинетического уравнения Власова, точнее, его свойства обратимости: уравнение (1.6) не меняется при подстановке

$$
t \mapsto-t, \quad v \mapsto-v
$$

В свою очередь это свойство - следствие обратимости исходных уравнений Ньютона. Напомним, что известное уравнение Больцмана, описывающее кинетику системы упруго сталкивающихся частиц, не допускает подстановку (1.9). В отличие от уравнения Власова, при выводе уравнения Больцмана делаются предположения, не совместимые с принципами классической механики (см. по этому поводу [7], [1], [9]).

Продолжим обсуждение уравнения Власова. Введем кинетическую энергию континуума

$$
T(t)=\frac{1}{2} \int \rho(x, v, t) v^{2} d x d v
$$

и суммарную потенциальную энергию взаимодействия

$$
V(t)=\frac{1}{2} \int \rho(x, v, t) \rho(y, u, t) K(x, y) d x d v d y d u
$$

в предположении, что $K(x, y)=K(y, x)$ для всех $x, y \in M$. Конечно, надо предполагать, что эти интегралы сходятся и являются гладкими функциями времени. Если опустить множитель $1 / 2$ в (1.10b), то потенциальная энергия взаимодействия каждой пары частиц будет учитываться дважды. В дискретном случае это обстоятельство было хорошо известно еще Лагранжу. В рассматриваемой модели "самодействие" отсутствует: каждая частица сама на себя не действует. Поэтому в формуле (1.10b) интегрирование по "диагонали" $\{x=y\}$ не проводится.

ТЕОРема 1.2. Для любого решения уравнения (1.6)

$$
T(t)+V(t)=\text { const. }
$$


Утверждение доказывается с использованием уравнения (1.6) и формулы Гаусса-Остроградского. Впрочем, этот факт очевиден с физической точки зрения.

Уравнения Власова имеют гамильтонову природу. Обсуждение этого факта с разных точек зрения можно найти в [9], [10]. Наверное, самый простой способ введения гамильтоновой структуры связан с использованием лагранжевых координат, которые "нумеруют" индивидуальные частицы континуума (см. [9; гл. 2]). С другой стороны, интеграл энергии (1.11) представлен в эйлеровых переменных, задающих место частиц в пространстве-времени.

В дальнейшем существенную роль будет играть еще один пример обобщенного кинетического уравнения:

$$
\begin{gathered}
\frac{\partial \rho}{\partial t}+\sum_{i=1}^{n}\left(\frac{\partial \rho}{\partial x_{i}} \frac{\partial \Psi}{\partial y_{i}}-\frac{\partial \rho}{\partial y_{i}} \frac{\partial \Psi}{\partial x_{i}}\right)=0 \\
\Psi(x, y, t)=\int K\left(x, y ; x^{\prime}, y^{\prime}\right) \rho\left(x^{\prime}, y^{\prime}, t\right) d^{n} x^{\prime} d^{n} y^{\prime}
\end{gathered}
$$

Здесь $x=\left(x_{1}, \ldots, x_{n}\right), y=\left(y_{1}, \ldots, y_{n}\right)$. Уравнение (1.12) будем называть $к u$ нетическим уравнением Гамильтона; В.П. Маслов называет его уравнением Власова-Лиувилля [10].

Tеорема 1.3. Eсли $K\left(x, y ; x^{\prime}, y^{\prime}\right)=K\left(x^{\prime}, y^{\prime} ; x, y\right)$, mo

$$
H=\frac{1}{2} \int K\left(x, y ; x^{\prime}, y^{\prime}\right) \rho(x, y, t) \rho\left(x^{\prime}, y^{\prime}, t\right) d^{n} x d^{n} y d^{n} x^{\prime} d^{n} y^{\prime}=\text { const. }
$$

Таким образом, в "стационарном" случае, когда ядро $K$ не зависит явно от времени, гамильтониан $H$ ("полная энергия" системы) является первым интегралом уравнения (1.12).

\section{§ 2. Динамика континуума осцилляторов}

В качестве примера рассмотрим континуум частиц в $m$-мерном евклидовом пространстве $E^{m}=\{x\}$, упруго притягивающихся друг к другу. В этом случае плотность потенциала $K(x, y)=W(|x-y|)$ равна

$$
\frac{k}{2}|x-y|^{2}=\frac{k}{2} \sum\left(x_{i}-y_{i}\right)^{2},
$$

где $k$ - коэффициент упругости. Согласно (1.6),

$$
F=-\int k(x-y) \rho(y, u, t) d y d u=-k(x-\xi),
$$

где

$$
\xi(t)=\int x \rho(x, v, t) d x d v
$$

- радиус-вектор центра масс системы. 
Покажем сначала, что $\ddot{\xi}=0$; т. е. центр масс движется прямолинейно и равномерно. Этот вывод, конечно, справедлив для любого потенциала взаимодействия, зависящего лишь от расстояния между частицами. Подсчитаем скорость центра масс:

$$
\dot{\xi}=-\int x\left[\left(\frac{\partial \rho}{\partial x}, v\right)-\left(\frac{\partial \rho}{\partial v}, \frac{\partial}{\partial x} \int W \rho(y, u, t) d y d u\right)\right] d x d v=\int v \rho d x d v
$$

Здесь использовалась формула Ньютона-Лейбница и естественное предположение, что $\rho \rightarrow 0$ при $x_{j} \rightarrow \pm \infty$ и $v_{j} \rightarrow \pm \infty$.

Далее,

$$
\ddot{\xi}=-\int v\left[\left(\frac{\partial \rho}{\partial x}, v\right)-\left(\frac{\partial \rho}{\partial v}, \frac{\partial}{\partial x} \int W \rho d y d u\right)\right] d x d v=0
$$

по формуле Гаусса-Остроградского, поскольку интеграл

$$
\int W \rho d y d u
$$

не зависит от $v$.

Итак, $\xi(t)=\alpha t+\beta$, где $\alpha$ и $\beta$ - постоянные векторы. Введем новые переменные

$$
z=x-\xi, \quad w=v-\alpha
$$

и положим

$$
\tilde{\rho}(z, w, t)=\rho(z+\xi, w+\alpha, t) .
$$

С учетом формулы (2.1) в новых переменных уравнение Власова (1.6) принимает вид

$$
\frac{\partial \tilde{\rho}}{\partial t}+\left(\frac{\partial \tilde{\rho}}{\partial z}, w\right)-\left(\frac{\partial \tilde{\rho}}{\partial w}, k z\right)=0
$$

Это - обычное уравнение Лиувилля для гармонического осциллятора. Оно легко решается переходом к переменным действие-угол.

Стационарные решения уравнения (2.2) - это функции от полной энергии осциллятора

$$
\frac{w^{2}+k z^{2}}{2}
$$

Нестационарные решения (2.2) как функции времени осциллируют и поэтому не имеют обычного предела при $t \rightarrow \pm \infty$. Однако они сходятся по Чезаро к стационарным решениям.

Задача о системе гармонических осцилляторов решена в [9; гл. 2] с использованием лагранжевых переменных. Показано, что все частицы колеблются около центра масс системы с одной и той же частотой. Однако нас интересует не динамика отдельных частиц, а эволюция их распределения. Конечно, в общем случае не приходится рассчитывать на явное сведение уравнения Власова к уравнению Лиувилля. 


\section{§ 3. Уравнение вихря 2D-гидродинамики как кинетическое уравнение}

Рассмотрим плоскопараллельное течение идеальной однородной жидкости в потенциальном силовом поле. Пусть $x, y$ - декартовы координаты в плоскости течения, $u, v$ - компоненты скорости частиц жидкости (они зависят от $x, y$ и времени $t$ ). Уравнения движения можно представить в виде уравнений Ламба

$$
\frac{\partial u}{\partial t}-\omega v=-\frac{\partial f}{\partial x}, \quad \frac{\partial v}{\partial t}+\omega u=-\frac{\partial f}{\partial y},
$$

где

$$
\omega=\frac{\partial v}{\partial x}-\frac{\partial u}{\partial y}
$$

- вихрь, а $f$ - функция Бернулли.

Из этих уравнений с учетом несжимаемости однородной жидкости $(\partial u / \partial x+$ $\partial v / \partial y=0)$ легко выводится уравнение, описывающее изменение вихря:

$$
\frac{\partial \omega}{\partial t}+\frac{\partial \omega}{\partial x} u+\frac{\partial \omega}{\partial y} v=0 .
$$

Таким образом, вихрь $\omega$ является первым интегралом дифференциальных уравнений

$$
\dot{x}=u(x, y, t), \quad \dot{y}=v(x, y, t),
$$

описывающих движение частиц жидкости. Отсюда вытекает, в частности, классическая теорема Гельмгольца-Томсона о вмороженности вихрей в плоскопараллельный поток идеальной жидкости. Как следствие получаем, что если

$$
\left.\omega\right|_{t=0} \geqslant 0
$$

то $\omega \geqslant 0$ при всех значениях $t$.

Уравнение (3.1) имеет вид статистического уравнения Лиувилля для неавтономной системы (3.2), поток которой сохраняет стандартную меру $d x d y$. Ввиду условия несжимаемости, уравнение (3.2) имеет гамильтонову форму

$$
u=-\frac{\partial \psi}{\partial y}, \quad v=\frac{\partial \psi}{\partial x},
$$

где $\psi(x, y, t)$ - функция тока. Таким образом, уравнение вихря - это уравнение Лиувилля гамильтоновой системы, которая, вообще говоря, неавтономная.

Укажем одно из следствий. Пусть $G$ - суммируемая функция одной переменной. Тогда интеграл

$$
\iint_{-\infty}^{\infty} G(\omega(x, y, t)) d x d y
$$

не зависит от времени (в предположении, что этот интеграл сходится). Другими словами, он будет интегральным инвариантом системы дифференциальных уравнений (3.2). В частности, моменты всех порядков - константы. Последнее 
обстоятельство является существенным пунктом доказательства теорем существования и единственности в плоской гидродинамике идеальной жидкости.

Покажем, что уравнение вихря (3.1) можно представить в виде стационарного кинетического уравнения Гамильтона (1.12). Действительно, это уравнение записывается в виде

$$
\frac{\partial \omega}{\partial t}-\frac{\partial \omega}{\partial x} \frac{\partial \psi}{\partial y}+\frac{\partial \omega}{\partial y} \frac{\partial \psi}{\partial x}=0
$$

где

$$
\psi=\iint_{-\infty}^{\infty} K\left(x, y ; x^{\prime}, y^{\prime}\right) \omega\left(x^{\prime}, y^{\prime}, t\right) d x^{\prime} d y^{\prime}
$$

- функция тока,

$$
K=\frac{1}{2 \pi} \ln \sqrt{\left(x-x^{\prime}\right)^{2}+\left(y-y^{\prime}\right)^{2}}
$$

- ядро линейного оператора - “парный потенциал взаимодействия". Функция $K$ совпадает с гамильтонианом, описывающим движение частиц жидкости в поле вихря с единичной интенсивностью.

Уравнение (3.3) (как и уравнение Лиувилля) есть статистическое уравнение. Пусть $\omega \geqslant 0$ при $t=0$ и выполнено условие нормировки:

$$
\left.\iint \omega\right|_{t=0} d \mu=1
$$

где $d \mu=d x d y$ - инвариантная “мера Лиувилля". Тогда в каждый момент времени выполнено неравенство $\omega \geqslant 0$,

$$
\iint_{-\infty}^{\infty} \omega(x, y, t) d \mu=1
$$

и

$$
P_{t}(D)=\iint_{g^{t}(D)} \omega(x, y, t) d \mu=\text { const. }
$$

В последней формуле $D$ - измеримая область плоскости $\mathbb{R}^{2}=\{x, y\}, g^{t}$ - поток системы (3.2), а $P_{t}(D)$ - вероятность нахождения частицы жидкости в области $D$. Таким образом, мы имеем распределение вероятностей на плоскости, которое согласовано с течением жидкости.

Подчеркнем, что время в уравнение (3.3) входит лишь через зависимость вихря от $t$.

\section{§4. Классические и обобщенные решения}

При наличии сингулярностей у ядра $K=\left\{K_{i}\right\}$ вопрос о существовании и единственности решений обобщенного кинетического уравнения Власова (1.1) является нетривиальной и пока нерешенной задачей. Чтобы представить возникающие здесь трудности, вспомним, что кинетическое уравнение Гамильтона (3.3) с логарифмическим ядром (3.4) эквивалентно уравнению вихря плоских течений идеальной баротропной жидкости. Таким образом, задача о существовании и единственности решений этого уравнения с классическими 
начальными условиями эквивалентна задаче о разрешимости гидродинамических уравнений Эйлера на плоскости. В двумерном случае регулярность решений при всех $t>0$ доказана Э. Гёльдером [11] и В. Волибнером [12]. Для трехмерных течений идеальной жидкости такого результата пока нет (см. [13], [14]). По-видимому, результаты Гёльдера и Волибнера можно перенести на многомерные кинетические уравнения Гамильтона (1.12).

Для гладких ядер (когда функции $K_{1}, \ldots, K_{n}$ бесконечно дифференцируемы) с некоторыми естественными ограничениями обобщенные кинетические уравнения Власова всегда разрешимы. Справедлива следующая теорема.

Теорема 4.1. Пусть $M$ замкнуто, функиии $K_{1}, \ldots, K_{n}$ гладкие и при $t=0$ плотность $\rho$ имеет непрерывные производные до порядка $k$ включительно (т.е. из класса $\left.C^{k}(M)\right)$. Тогда уравнение (1.1) имеет единственное решение из класса $C^{k}$, определенное при всех значениях $t$.

Случай некомпактного $M$ мы обсудим чуть позже. Существование непрерывно дифференцируемых решений установлено в работах [10], [15], а единственность - в [10], [16]. Правда, в этих работах рассматривался случай $M=\mathbb{R}^{n}$ и были сделаны некоторые дополнительные предположения.

Нас будут также интересовать решения уравнения (1.1), принадлежащие классам $L_{p}(M, d \nu), p \geqslant 1$, которые следует понимать в обобщенном (слабом) смысле. Пусть $D(M)$ - пространство Шварца вещественных бесконечно дифференцируемых функций с компактным носителем; если $M$ замкнуто, то, очевидно, $D(M)=C^{\infty}(M)$. Будем говорить, что $\rho_{t}$ является слабым решением уравнения (1.5), если все функции

$$
\int_{M} \rho_{t} h d \nu, \quad h \in D(M),
$$

дифференцируемы по времени $t$ и

$$
\frac{\partial}{\partial t} \int_{M} \rho_{t} h d \nu-\sum_{i} \int_{M} \rho_{t} \frac{\partial h v_{i}}{\partial x_{i}} d \nu=0 .
$$

Уравнение (4.1) получается из (1.5) умножением на “пробную" функцию $h$ и интегрированием по частям. Поскольку ядра $\left\{K_{i}\right\}$ - гладкие функции, то $v=\left(v_{1}, \ldots, v_{n}\right)$ - гладкое поле на $M$ :

$$
\frac{\partial v_{i}}{\partial x_{i}}=\int \frac{\partial K_{i}(x, y)}{\partial x_{i}} \rho(y, t) d^{n} y .
$$

Теорема 4.2. Пусть $M$ замкнуто, функиии $K_{j}(1 \leqslant j \leqslant n)$ гладкие $и$ $\rho \in L_{p}$ nри $t=0$. Тогда уравнение (1.1) имеет единственное (с точностью до значений на множестве нулевой меры) слабое решение из класса $L_{p}(M, d \nu)$, определенное при всех $t \in \mathbb{R}$.

Это утверждение доказывается методом работы [16], в которой разобран случай, когда $M=\mathbb{R}^{n}$ и $p=1$.

Сделаем несколько замечаний по поводу теорем 4.1 и 4.2, раскрывающих один из аспектов свойства "самосогласованности" уравнения (1.1). Если ядра 
$K_{1}, \ldots, K_{n}$ - ограниченные бесконечно дифференцируемые функции, а плотность $\rho_{t}$ суммируема при всех $t$, то компоненты $v_{i}$ векторного поля из $(1.3)$ также будут гладко зависеть от точки $x \in M$. Это вытекает из второй формулы (1.1) и теоремы о дифференцировании интеграла по параметру. Следовательно, если $\rho$ - слабое решение уравнения (1.1), то поле $v$ гладкое и поэтому система обыкновенных дифференциальных уравнений (1.3) однозначно разрешима. Пусть $\left\{g^{t}\right\}-$ ее поток:

$$
x\left(t, x_{0}\right)=g^{t}\left(x_{0}\right),
$$

где $x\left(t, x_{0}\right)$ - решение $(1.3)$ с условием Коши $x\left(0, x_{0}\right)=x_{0}$. Ввиду неавтономности системы (1.3), семейство преобразований $\left\{g^{t}\right\}$ в общем случае не является группой, хотя каждое из преобразований $g^{t}$ обратимо. Положим $g^{-t}=\left(g^{t}\right)^{-1}$. Тогда

$$
x_{0}=g^{-t}(x) .
$$

Согласно предположению (1.2), $\operatorname{div} v=0$. Следовательно, первое уравнение (1.1) можно записать в виде

$$
\frac{\partial \rho}{\partial t}+\sum \frac{\partial \rho}{\partial x_{i}} v_{i}=0 .
$$

Это означает, что $\rho=d \mu / d \nu$ - первый интеграл системы (1.3).

Из (4.2) и (4.3) вытекает простая, но важная формула

$$
\rho_{t}(x)=\rho_{0}\left(g^{-t}(x)\right) .
$$

Действительно,

$$
\rho_{t}\left(g^{t}\left(x_{0}\right)\right)=\rho_{0}\left(x_{0}\right)=\rho_{0}\left(g^{-t}(x)\right) .
$$

Формула (4.4) справедлива и для уравнения Лиувилля (когда ядра $K_{i}$ не зависят от второго аргумента). Правда, в этом случае уравнения (1.3) будут автономными и однопараметрическое семейство преобразований $\left\{g^{t}\right\}$ является группой.

Если $M$ замкнуто, то все решения системы (1.3) определены на всей оси времени $\mathbb{R}=\{t\}$. Кроме того, при всех $t$ преобразования $g^{t}$ и $g^{-t}$ будут диффеоморфизмами $M$, сохраняющими меру $d \nu$. Значит, если $\rho_{0} \in C^{m}(M)$, то при всех $t$ функция $\rho_{t}$ также $m$ раз непрерывно дифференцируема. Это замечание, конечно, справедливо и при $m=\infty$. Аналогично, если $\rho_{0} \in L_{p}(1 \leqslant p \leqslant \infty)$, то функция $x \mapsto \rho_{t}(x)$ также принадлежит $L_{p}$. Таким образом, теоремы 4.1 и 4.2 выводятся из факта существования и единственности слабого решения исходного уравнения (1.1).

Если же $M$ некомпактно, то может так случиться, что поток $\left\{g^{t}\right\}$ системы (1.3) определен не при всех значениях $t$. В этом случае заключения теорем 4.1 и 4.2, конечно, не справедливы. Здесь нужны дополнительные предположения. Например, пусть $M=\mathbb{R}^{n}$ и все ядра $K_{1}, \ldots, K_{n}$ ограничены. Тогда поле $v$ будет иметь ограниченные компоненты и, следовательно, решения системы (1.3) продолжаются на всю временну́ю ось.

Конечно, вопросы однозначной разрешимости нелинейных уравнений имеют существенное значение. Однако смысл нашей работы вовсе не в этом. Поэтому 
мы будем молчаливо предполагать справедливость выводов теорем 4.1 и 4.2 и в случае, когда $M$ незамкнуто.

\section{§ 5. Кинетическое уравнение как динамическая система}

Введем однопараметрическое семейство операторов

$$
G^{t}: \rho_{0} \mapsto \rho_{t}
$$

Согласно теоремам 4.1 и 4.2, эти операторы переводят функциональные пространства $C^{m}(M)(0 \leqslant m \leqslant \infty)$ и $L_{p}\left(M, d^{n} x\right)(1 \leqslant p \leqslant \infty)$ в себя. Однако они, как правило, нелинейные (хотя оператор $G^{0}$ единичный и поэтому линейный).

Теорема 5.1. Семейство операторов $\left\{G^{t}\right\}, t \in \mathbb{R}$, является однопараметрической группой:

(a) $G^{0}-$ тождественное преобразование;

(b) $\left(G^{t}\right)^{-1}=G^{-t}$ для всех $t$;

(c) $G^{t_{2}}\left(G^{t_{1}}\right)=G^{t_{1}+t_{2}}$ для всех $t_{1}, t_{2}$.

Это утверждение - следствие обобщенного кинетического уравнения (1.1) и предположения о независимости ядер $\left\{K_{i}\right\}$ от времени. Действительно, пусть $\rho_{t}$ и $\tilde{\rho}_{t}-$ решения уравнения (1.1) с одним и тем же начальным условием, но при $t=0$ и $t=\tau$ соответственно. Тогда (с учетом теоремы единственности)

$$
\rho_{t}=\tilde{\rho}_{t+\tau}
$$

для всех $t \in \mathbb{R}$. Из этого равенства легко выводятся два последних заключения теоремы 5.1.

Из теоремы 1.1 вытекает

Теорема 5.2. Оператори $G^{t}$ переводят сфери

$$
\|\rho\|_{L_{p}}=\left[\int_{M}|\rho(x)|^{p} d^{n} x\right]^{\frac{1}{p}}=\mathrm{const}
$$

из $L_{p}(p<\infty)$ в себя.

Отметим еще, что $G^{t}$ сохраняют также равномерную норму в $C^{0}(M)$ :

$$
\|\rho\|_{C^{0}}=\sup _{x \in M}|\rho(x)| .
$$

Если операторы $G^{t}$ были бы линейными, то они были бы изометриями $L_{p}$ :

$$
\left\|\rho_{t}^{(1)}-\rho_{t}^{(2)}\right\|=\mathrm{const}
$$

для любых двух решений уравнения (1.1). Для обычного уравнения Лиувилля операторы $G^{t}$, очевидно, линейные; соответствующие операторы $L_{2} \rightarrow L_{2}$ обычно называются операторами Купмана. Свойства линейности и изометричности позволяют доказать статистическую эргодическую теорему фон Неймана: для любой функции $\rho \in L_{2}$ найдется $\bar{\rho} \in L_{2}$ такая, что

$$
\lim _{\tau \rightarrow \infty}\left\|\frac{1}{\tau} \int_{0}^{\tau} G^{t}(\rho) d t-\bar{\rho}\right\|_{L_{2}}=0
$$

и $G^{t} \bar{\rho}=\bar{\rho}$ для всех $t \in \mathbb{R}$. 
Очевидно, что из сходимости в $L_{2}$ вытекает слабая сходимость по Чезаро:

$$
\lim _{\tau \rightarrow \infty} \frac{1}{\tau} \int_{0}^{\tau} \int_{M} \rho_{t} \varphi d^{n} x d t=\int_{M} \bar{\rho} \varphi d^{n} x
$$

для любой “пробной” функции $\varphi \in L_{2}$. В частности, если $M$ компактно, то

$$
\int_{M} \bar{\rho} d^{n} x=\int_{M} \rho_{0} d^{n} x
$$

Это сразу же вытекает из (5.2), если положить $\varphi(x)=1$ и воспользоваться (1.4). Таким образом, если мера $\rho_{t} d^{n} x$ вероятностная, то ее слабый предел $\bar{\rho} d^{n} x$ также будет вероятностной мерой.

Последнее наблюдение имеет существенное значение для понимания задачи о стремлении континуума взаимодействующих частиц к состоянию статистического равновесия. Равенство (5.2) можно считать определением статистического равновесия. Такой подход естествен с точки зрения обоснования термодинамики - перехода к макроскопическому описанию эволюции динамической системы, поскольку плотность вероятностной меры "существует" не сама по себе, а проявляется при вычислении средних значений динамических величин. С этой точки зрения пробным функциям соответствуют физические устройства, измеряющие средние (наиболее вероятные) величины.

Подход к обоснованию термодинамики, основанный на анализе слабых пределов решений уравнения Лиувилля (которое управляет эволюцией ансамблей Гиббса), изложен в книгах [2], [3]. В этом случае слабый предел по Чезаро всегда существует и совпадает с биркгофовским средним начальной плотности распределения вероятностей. В ряде важных для приложений случаев (например, гамильтоновы системы с однородным потенциалом, степень которого не равна двум, а также системы биллиардного типа) сходимость по Чезаро в (5.2) можно заменить обычной сходимостью [17].

K сожалению, ввиду нелинейности уравнения (1.1), указанный выше подход непосредственно не применим. Однако можно попытаться рассуждать по-другому, рассматривая семейство операторов $\left\{G^{t}\right\}, t \in \mathbb{R}$, как нелинейную динамическую систему с фазовым пространством $L_{2}\left(M, d^{n} x\right)$. Конечно, $L_{2}$ можно заменить более общим пространством $L_{p}$ или же $C^{m}(m \geqslant 0)$. Но проще всего начать с рассмотрения системы в $L_{2}$, которое имеет естественную структуру гильбертова пространства.

Будем исходить из следующей гипотезы:

- в $L_{2}$ имеется счетно-аддитивная мера $\Lambda$, инвариантная относительно фазового потока $\left\{G^{t}\right\}$.

Это предположение, конечно, не самоочевидно и требует доказательства. На самом деле нам было бы достаточно иметь такую меру на единичной сфере

$$
\|\rho\|_{L_{2}} \leqslant 1
$$

которая инвариантна относительно преобразований $\left\{G^{t}\right\}$. Основная трудность построения инвариантной меры связана с некомпактностью этой сферы.

Классическая теорема Крылова-Боголюбова гарантирует существование инвариантной конечной счетно-аддитивной меры для любой динамической 
системы на компактном пространстве. Однако, ввиду универсальности этой теоремы, инвариантная мера может оказаться весьма нерегулярной. В качестве примера рассмотрим простую систему

$$
\dot{x}=-\sin x
$$

на окружности $\mathbb{T}^{1}=\{x \bmod 2 \pi\}$. Она имеет два равновесия: $x=0$ и $x=\pi$. Первое из них асимптотически устойчиво, а второе, наоборот, неустойчиво. Система (5.3) имеет вероятностную инвариантную меру $\Lambda: \Lambda(A)=1$, если $0 \in A$, и $\Lambda(A)=0$, если $0 \in \mathbb{T}^{1} \backslash A$. Эта мера счетно-аддитивна, но сингулярна по отношению к обычной мере Лебега на $\mathbb{T}^{1}$; ее плотность - дельта-функция Дирака.

Однако в ряде случаев удается обосновать сформулированную выше гипотезу и установить наличие “хорошей” инвариантной меры. Вспомним кинетическое уравнение Гамильтона (1.12) с симметричным ядром $K$ и рассмотрим линейный оператор

$$
\rho(x, y) \mapsto \int K\left(x, y ; x^{\prime}, y^{\prime}\right) \rho\left(x^{\prime}, y^{\prime}\right) d^{n} x^{\prime} d^{n} y^{\prime} .
$$

Если этот оператор является оператором Гилъберта-Шмидта (т. е. интеграл от $K^{2}$ по $M \times M$ конечен), то гамильтониан (1.13) (полная энергия) принимает конечные значения при квадратично суммируемой плотности $\rho$. В этом случае все собственные значения оператора (5.4) - вещественные положительные числа и сумма их квадратов конечна. Если же ряд, составленный из собственных чисел (с учетом их кратностей) абсолютно сходится, то оператор (5.4) будет ядерным оператором.

ТеОрема 5.3. Если оператор (5.4) ядерный, то динамическал система $\left\{G^{t}\right\}, t \in \mathbb{R}$, допускает инвариантную гауссовскую меру в $L^{2}(M, d \nu)$.

Действительно, пусть $\Lambda$ - мера в $L^{2}(M, d \nu), d \nu=d^{n} x d^{n} y$, с характеристическим функционалом (преобразование Фурье меры $\Lambda$ )

$$
\widehat{\Lambda}=e^{-H},
$$

где $H$ - "гамильтониан" (1.13). Поскольку оператор (5.4) ядерный, то такая мера существует и является гауссовской (см., например, [18]); ее среднее равно нулю, а ковариационный оператор совпадает с оператором (5.4). Согласно теореме 1.3 , характеристический функционал меры $\Lambda$ не зависит от времени. Следовательно, эта мера инвариантна относительно преобразований $G^{t}, t \in \mathbb{R}$. Что и требовалось.

Напомним, что гауссовская мера конечна: $\Lambda\left(L_{2}\right)<\infty$. K сожалению, кинетическое уравнение Гамильтона с логарифмическим ядром (описывающее плоское течение идеальной жидкости) не удовлетворяет условиям теоремы 5.3. Вообще, вопрос о наличии регулярной инвариантной меры для общего уравнения (1.1) упирается в содержательные задачи анализа. Поучительное обсуждение круга вопросов, связанного с введением меры в бесконечномерных пространствах, содержится в недавней работе А. М. Вершика [19]. 


\section{§6. Слабые пределы решений обобщенного кинетического уравнения}

Наша цель - обосновать слабую сходимость по Чезаро - соотношение (5.2). В частности, это позволит нам сделать естественный переход от микро- к макроописанию континуумов взаимодействующих частиц и решить задачу о стремлении системы к состоянию статистического равновесия.

Будем исходить из предположения о наличии счетно-аддитивной инвариантной меры $\Lambda$ динамической системы $\left\{G^{t}\right\}$. Это дает нам возможность применить индивидуальную эргодическую теорему Биркгофа-Хинчина к непреръвной функции на $L_{2}(M, d \nu)$, заданной линейным функционалом

$$
\rho \mapsto \int_{M} \varphi \rho d^{n} x
$$

Значение этой функции в текущий момент времени определяется той же формулой, только $\rho$ надо заменить на $\rho_{t}=G^{t}(\rho)$.

Прежде всего надо убедиться в том, что функция (6.1) интегрируема по мере $\Lambda$ на пространстве $L_{2}$. Этот вопрос, конечно, не самоочевидный. Но если $\Lambda$ - гауссовская мера, то любой непрерывный линейный функционал будет, конечно, интегрируемой функцией.

Укажем еще один простой случай, когда вопрос о суммируемости решается положительно. Рассмотрим функционал (6.1) на пространстве $L_{1}\left(M, d^{n} x\right)$; "пробную" функцию $\varphi$ следует выбрать существенно ограниченной (из $L_{\infty}$ ). Если инвариантная мера $\Lambda$, заданная на сфере

$$
S=\left\{\rho: \int_{M}|\rho(x)| d^{n} x=1\right\},
$$

конечна, то линейный функционал (6.1) будет непрерывной и ограниченной функцией на $S$. В частности, (6.1) - интегрируемая функция по конечной мере на $S$.

Согласно индивидуальной эргодической теореме (с уточнением КрыловаБоголюбова),

$$
\lim _{\tau \rightarrow \infty} \frac{1}{\tau} \int_{0}^{\tau} \int_{M} \varphi \rho_{t} d^{n} x d t
$$

существует для почти всех (по мере $\Lambda$ ) начальных плотностей $\rho_{0}$. Уточнение Крылова-Боголюбова состоит в том, что чезаровское среднее непрерывной функции существует на множестве полной меры, которая зависит лишь от структуры динамической системы, но не от усредняемой функции (см., например, [20; гл. VI]). В нашем случае исключительное множество начальных данных $\left\{\rho_{0}\right\}$ не зависит от выбора функции $\varphi$ в (6.1).

Результат о существовании предела (6.2) можно интерпретировать следующим образом. Так как

$$
\left(\int \varphi \rho_{t} d^{n} x\right)^{2} \leqslant \int \varphi^{2} d^{n} x \int \rho_{t}^{2} d^{n} x
$$


и второй сомножитель справа не зависит от времени (следствие 1.2 теоремы 1.1), то функция

$$
t \mapsto \int_{M} \varphi \rho_{t} d^{n} x
$$

ограничена. Однако она осциллирует и поэтому не имеет предела (в обычном смысле) при $t \rightarrow \pm \infty$. Тем не менее, при определенных условиях функция (6.3) сходится по Чезаро. Напомним, что метод Чезаро включает обычное определение сходимости. Метод Чезаро можно заменить любым более сильным методом суммирования (например, методом Абеля). Не исключено, что при такой замене можно ослабить условия на уравнение (1.1), при которых сходятся средние значения динамических величин (6.3).

Чезаровское среднее (6.2) - положителъный линейный функционал на пространстве пробных функций: если $\varphi \geqslant 0$, то среднее также неотрицательно (поскольку $\left.\rho_{t} \geqslant 0\right)$. При некоторых дополнительных предположениях его можно представить в виде интеграла

$$
\int_{M} \varphi(x) d \bar{\mu},
$$

где $\bar{\mu}$ - некоторая мера на $M$. Эта мера и будет слабым пределом по Чезаро исходной меры

$$
d \mu_{t}=\rho_{t}(x) d^{n} x
$$

при $t \rightarrow \pm \infty$. Отметим, что предельная мера также будет вероятностной:

$$
\int_{M} d \bar{\mu}=1 .
$$

Для доказательства достаточно положить $\varphi(x)=1, x \in M$.

Эта идея нуждается в уточнении. Пусть $M^{n}=\{x\}$ - гладкое замкнутое многообразие с лебеговой мерой $d \nu=d^{n} x$, а начальная плотность $\rho_{0}$ - суммируемая функция (из $L_{1}(M, d \nu)$ ). Как уже было сказано в $\S 4$, тем же свойством обладает функция $\rho_{t}$ при всех $t \in \mathbb{R}$. В качестве динамических величин $\{\varphi\}$ в формуле (6.2) следует брать существенно ограниченные функции (из $L_{\infty}(M, d \nu)$ ).

Теорема 6.1. Предположим, что в $L_{1}(M, d \nu)$ имеется счетно-аддитивная мера $\Lambda$, инвариантная относительно группь $\left\{G^{t}\right\}$. Тогда для почти всех (по мере $\Lambda$ ) началъных условий $\rho_{0} \in L_{1}$ существует слабый предел $\bar{\rho}$ (по Чезаро) решения $\rho_{t}$ уравнения (1.1), который является

1) функиией из $L_{1}(M, d \nu)$,

2) обобщенным стационарным решением уравнения (1.1),

3) плотностъю вероятностной меры (т.е. $\bar{\rho} \geqslant 0$ и выполнено (6.5)).

Как уже отмечалось в $\S 5$, для более простого уравнения Лиувилля (когда в уравнении (1.1) ядра не зависят от второй переменной) слабый предел по Чезаро всегда существует и совпадает с биркгофовским средним начальной плотности $\rho_{0}$. В этом случае свойства 1$)-3$ ) слабого предела из теоремы 6.1 совпадают с хорошо известными из эргодической теории свойствами биркгофовских 
средних неотрицательной суммируемой функции. Только заключение 2) теоремы 6.1 в эргодической теории обычно формулируется по-другому: биркгофовское среднее - первый интеграл системы (1.3), которая в случае уравнения Лиувилля будет автономной. Однако эти формулировки на самом деле эквивалентны.

В связи с теоремой 6.1 возникает интересная задача: верно ли, что если $\rho_{0} \in$ $L_{p}(M, d \nu)(p>1)$, то слабый предел $\bar{\rho}$ также будет функцией из $L_{p} ?$ В эргодической теории положительный ответ известен достаточно давно (см. [21], [22]).

Укажем основные моменты доказательства теоремы 6.1. Пусть $A-\nu$-измеримое подмножество замкнутого многообразия и $\varphi=\varphi_{A}-$ его характеристическая функция. Ясно, что $\varphi_{A} \in L_{\infty}(M, d \nu)$ и

$$
\int_{M} \varphi_{A} \rho_{t} d \nu=\int_{A} \rho_{t} d \nu=\int_{g^{-t}(A)} \rho_{0} d \nu
$$

причем $\nu\left(g^{-t}(A)\right)=\nu(A)$ при всех значениях $t$. Чезаровское среднее $(6.2)$ есть функция множества, определенная на $\sigma$-алгебре всех $\nu$-измеримых множеств многообразия $M$. Эта функция есть вероятностная мера, которую мы выше обозначили $\bar{\mu}$. Из формул (6.2) и (6.6) вытекает, что мера $\bar{\mu}$ абсолютно непрерывна относительно меры $\nu$ : если $\nu(A)=0$, то, очевидно, $\bar{\mu}(A)=0$. Так как $\bar{\mu}(M)<\infty$, то из теоремы Радона-Никодима вытекает существование $\nu$-интегрируемой плотности $d \bar{\mu} / d \nu=\bar{\rho}$. Эта функция и будет слабым пределом решения $\rho_{t}$ уравнения (1.1) при $t \rightarrow \infty$. Подчеркнем, что слабые пределы $\rho_{t}$ при $t \rightarrow+\infty$ и $t \rightarrow-\infty$, конечно, совпадают.

Докажем теперь заключение 2). Действительно, для любой существенно ограниченной измеримой функции $\varphi$ на замкнутом многообразии $M$ значение интеграла

$$
\int_{M} G^{t}(\bar{\rho}) \varphi d \nu
$$

не зависит от времени. Следовательно, $G^{t}(\bar{\rho})$ также не зависит от $t$. Это обстоятельство можно выразить формулой:

$$
\bar{\rho}_{t}=G^{t}\left(\bar{\rho}_{0}\right)=\bar{\rho}
$$

для всех $t$ (где, конечно, $\bar{\rho}_{0}=\bar{\rho}$ ). Таким образом, постоянная функция $t \mapsto$ $G^{t}(\bar{\rho})=\bar{\rho}$ задает стационарное (в общем случае обобщенное) решение уравнения (1.1).

Наконец, заключение 3) теоремы 6.1 - непосредственное следствие формулы (6.5).

Таким образом, согласно теореме 6.1, задача о слабых пределах решений обобщенного кинетического уравнения Власова сводится к задаче об инвариантных мерах в соответствующих функциональных пространствах.

Векторному полю $v=\left(v_{1}, \ldots, v_{n}\right)$ из $(1.1)$ (вообще говоря, зависящему от времени) полезно сопоставить среднее поле $\bar{v}=\left(\bar{v}_{1}, \ldots, \bar{v}_{n}\right)$, где

$$
\bar{v}_{i}=\lim _{\tau \rightarrow \infty} \frac{1}{\tau} \int_{0}^{\tau}\left(\int_{M} K_{i}(x, y) \rho_{t}(y) d^{n} y\right) d t=\int_{M} K_{i}(x, y) \bar{\rho}(y) d^{n} y .
$$


Если ядра $\left\{K_{i}\right\}$ - гладкие функции, то поле $\bar{v}$ также будет гладким векторным полем на $M$. Ввиду предположения (1.2), фазовый поток автономной системы

$$
\dot{x}=\bar{v}(x), \quad x \in M,
$$

сохраняет форму объема $d^{n} x$. Кроме того, неотрицательная суммируемая функция $\bar{\rho}: M \rightarrow \mathbb{R}$ - первый интеграл (инвариант) этой системы. В частности, если система (6.7) эргодическая, то

$$
\bar{\rho}=\frac{1}{\nu(M)}, \quad \nu(M)=\int_{M} d \nu,
$$

почти всюду.

Таким образом, равновесные состояния для обобщенных кинетических уравнений определяются так же, как и в теории ансамблей Гиббса. Однако задача о приближении системы к состоянию статистического равновесия здесь существенно сложнее.

Эти соображения можно применить к плоской гидродинамике идеальной жидкости; она описывается кинетическим уравнением Гамильтона (3.3). Если вихрь $\omega_{t}$ слабо сходится к интегрируемой функции $\bar{\omega}$, то нестационарное течение идеальной жидкости стремится "в среднем" к стационарному течению с вихрем $\bar{\omega}$. Его поле скоростей находится по известным формулам

$$
\begin{aligned}
& \bar{u}(x, y)=-\frac{1}{2 \pi} \iint \frac{\left(y-y^{\prime}\right) \bar{\omega}\left(x^{\prime}, y^{\prime}\right)}{\sqrt{\left(x-x^{\prime}\right)^{2}+\left(y-y^{\prime}\right)^{2}}} d x^{\prime} d y^{\prime}, \\
& \bar{v}(x, y)=\frac{1}{2 \pi} \iint \frac{\left(x-x^{\prime}\right) \bar{\omega}\left(x^{\prime}, y^{\prime}\right)}{\sqrt{\left(x-x^{\prime}\right)^{2}+\left(y-y^{\prime}\right)^{2}}} d x^{\prime} d y^{\prime} .
\end{aligned}
$$

Полезно отметить, что в этих интегральных формулах ядра являются существенно ограниченными функциями. Поэтому средние скорости корректно определены для любой завихренности $\omega$ из $L_{1}\left(\mathbb{R}^{2}, d x d y\right)$. Поле скоростей $(\bar{u}, \bar{v})$ следует отличать от “среднего поля", введенного в работах [23], [24].

На траекториях (линиях тока) стационарной системы

$$
\dot{x}=\bar{u}(x, y), \quad \dot{y}=\bar{v}(x, y)
$$

постоянна функция тока

$$
\bar{\Psi}=\iint K\left(x, y ; x^{\prime}, y^{\prime}\right) \bar{\omega}\left(x^{\prime}, y^{\prime}\right) d x^{\prime} d y^{\prime},
$$

где $K$ дается формулой (3.4). Ее линии уровня могут быть устроены весьма сложно (см., например, работу [25], где, в частности, обсуждаются геометрические свойства линий уровня случайно выбранной функции на плоскости). Однако в действительности функция $\bar{\Psi}$ не может быть произвольной. Дело в том, что $\bar{\omega}$ также является первым интегралом системы (6.8). Следовательно, функции $\bar{\Psi}$ и $\bar{\omega}$ зависимы: в гладком случае их якобиан тождественно равен нулю. Локально можно считать, что

$$
\bar{\omega}=F(\bar{\Psi}),
$$


где $F(\cdot)$ - функция одного переменного. Важный подкласс составляют стационарные течения, когда соотношение (6.9) выполнено в целом. Если предельное "среднее поле" (6.8) гладкое, то $\bar{\omega}=\Delta \bar{\Psi}$ и уравнение (6.9) можно записать в следующем виде:

$$
\Delta \bar{\Psi}=F(\bar{\Psi})
$$

Здесь $\Delta$ - оператор Лапласа на плоскости.

По аналогии с равновесной статистической механикой, в работе [26] предложено в качестве функции $F$ брать плотность канонического распределения Гиббса:

$$
F(\bar{\Psi})=\frac{e^{-\beta \bar{\Psi}}}{Z(\beta)} .
$$

Здесь $\beta$ - некоторый параметр, а нормировочный множитель $Z$ находится из соотношения

$$
\iint F(\bar{\Psi}(x, y)) d x d y=\iint \bar{\omega} d x d y=1
$$

В статистической механике $\beta$ обычно полагают равным $1 /(k T)$, где $k$ - постоянная Больцмана, а $T$ - абсолютная температура. Для некоторых стационарных течений параметр $\beta$ может быть отрицательным. Возможность существования равновесных состояний с отрицательной “температурой” была отмечена еще в классической работе Л. Онзагера [27], что вызвало оживленную дискуссию.

Вообще, для работ по плоской турбулентности характерно некритическое использование соотношений и методов классической статистической механики. В качестве примера приведем работу [28], в которой с использованием цепочек уравнений Боголюбова (Б-Б-Г-К-И иерархия уравнений) выводится необратимое кинетическое уравнение для системы большого числа точечных вихрей (аналог уравнения Больцмана). Однако следует иметь в виду, что по ходу вывода этого уравнения делается ряд дополнительных предположений, несовместимых с обратимой эволюцией системы вихрей.

Наш подход, намеченный в докладе [29], основан на других идеях. Для почти всех начальных завихренностей $\omega_{0}$ вихрь $\omega(t)$ слабо сходится при $t \rightarrow \pm \infty$ к предельной функции $\bar{\omega}$, которая описывает обобщенное стационарное течение. Эта функция удовлетворяет соотношению (6.9), которое в свою очередь эквивалентно уравнению второго порядка (6.10) на функцию тока с условием (6.11). Ввиду некомпактности области течения и логарифмической особенности у ядра (3.4), слабый предел $\bar{\omega}$ может оказаться негладкой функцией. В этом случае эту конструкцию, конечно, следует понимать в обобщенном смысле (как объяснено в §4).

\section{§ 7. Сингулярные распределения}

Описанная в предыдущем параграфе картина стремления решений обобщенного уравнения Власова к равновесному состоянию упирается в доказательство содержательной гипотезы о наличии инвариантной меры $\Lambda$ динамической сис- 
темы в $L_{2}$. Однако на физическом уровне строгости эту проблему легко обойти, если рассматривать конечномерные пространства решений этого уравнения с сингулярностями.

Итак, будем искать решения уравнения (1.1) в виде взвешенной суммы $\delta$-функций Дирака:

$$
\rho(x, t)=\sum_{j=1}^{N} \varkappa_{j} \delta\left(x_{1}-x_{1}^{(j)}(t)\right) \cdots \delta\left(x_{n}-x_{n}^{(j)}(t)\right) .
$$

Точки из $M^{n}$ с координатами

$$
\left(x_{1}^{(j)}(t), \ldots, x_{n}^{(j)}(t)\right)=X_{j}(t), \quad 1 \leqslant j \leqslant N,
$$

- сингулярности меры $d \mu_{t}=\rho(x, t) d^{n} x$ с плотностью (7.1). Числа $\varkappa_{j}$ можно назвать интенсивностями сингулярностей (7.2); поскольку $\rho \geqslant 0$, то все $\varkappa_{j}>0$. Оказывается, если (7.1) является обобщенным решением кинетического уравнения (1.1), то функции $t \mapsto X_{j}(t)$ удовлетворяют некоторой специальной конечномерной автономной системе обыкновенных дифференциальных уравнений с инвариантной счетно-аддитивной мерой.

ТЕорема 7.1. Сингулярности (7.2) удовлетворяют следующей системе дифберенциалъных уравнений:

$$
\begin{gathered}
\dot{x}_{i}^{(j)}=\sum_{k=1}^{N} \varkappa_{k} K_{i}\left(x_{1}^{(j)}, \ldots, x_{n}^{(j)} ; x_{1}^{(k)}, \ldots, x_{n}^{(k)}\right), \\
i=1, \ldots, n, \quad j=1, \ldots, N .
\end{gathered}
$$

Записывая в явном виде уравнения (7.3), мы предполагаем, что все сингулярности лежат в пределах одной карты на многообразии $M$. В противном случае (7.3) следует представить в инвариантном виде, как это сделано в [16] для обычного уравнения Власова. Впрочем, на замкнутом многообразии $M$ можно указать одну карту, которая покрывает почти все $M$. С другой стороны, если $M$ диффеоморфно прямому произведению $\mathbb{R}^{l} \times \mathbb{T}^{n-l}$, то можно ввести $n$ "глобальных" переменных, среди которых $l$ линейных и $n-l$ угловых.

Укажем схему доказательства теоремы 7.1. Согласно (1.1) и определению $\delta$-функции,

$$
v_{i}(x, t)=\sum_{k=1}^{N} \varkappa_{k} K_{i}\left(x, X_{k}(t)\right) .
$$

Далее, уравнение (4.1) с учетом соглашения (1.2) принимает вид:

$$
\frac{d}{d t} \sum_{j} \varkappa_{j} h\left(X_{j}\right)=\left.\sum_{i, j, k} \varkappa_{j} \varkappa_{k} \frac{\partial h}{\partial x_{i}}\right|_{x=X_{j}} K_{i}\left(X_{j}, X_{k}\right) .
$$

Левая часть равна

$$
\left.\sum_{i, j} \varkappa_{j} \frac{\partial h}{\partial x_{i}}\right|_{x=X_{j}} \dot{x}_{i}^{(j)}
$$


Ясно, что уравнение (7.5) (с учетом (7.6)) удовлетворяется для любой гладкой функции $h$, если скорости $\dot{x}_{i}^{(j)}$ заменить выражением (7.3). Однако нам надо доказать обратное утверждение, используя подходящий выбор функции $h$.

Продемонстрируем идею доказательства в простейшем нетривиальном случае, когда $n=1$. С учетом (7.6) уравнение (7.5) представим в следующем виде:

$$
\left.\sum_{j=1}^{N} \varkappa_{j} \xi_{j} \frac{\partial h}{\partial x}\right|_{x=X_{j}}=0
$$

где $\xi_{j}$ - разность между левой и правой частью (7.3). Положим последовательно $h(x)$ равным

$$
x, x^{2}, \ldots, x^{N} .
$$

Вне области, содержащей сингулярности $X_{1}, \ldots, X_{N}$, эти функции можно гладко продолжить до финитных функций. После подстановки (7.8) в (7.7) получим линейную однородную систему относительно $\varkappa_{1} \xi_{1}, \ldots, \varkappa_{N} \xi_{N}$ с определителем Вандермонда. В типичном случае, когда $X_{j} \neq X_{k}$ при $j \neq k$, определитель отличен от нуля. Следовательно, $\xi_{1}=\cdots=\xi_{N}=0$. По непрерывности эти равенства останутся справедливыми и при совпадении сингулярностей. Что и требовалось.

Как заметил сам Власов, уравнение (1.6) допускает точное обобщенное решение вида (7.1), причем сингулярности меры интерпретируются как частицы, а их интенсивности - как массы частиц (см. [30]). Уравнения эволюции сингулярностей совпадают с уравнениями Ньютона для системы взаимодействующих частиц. Более удивительным выглядит теорема Боголюбова [31] о наличии сингулярных решений вида (7.1) у кинетического уравнения Больцмана-Энскога для упруго сталкивающихся шаров. Это уравнение (являющееся модификацией классического уравнения Больцмана) обычно рассматривается как приближение малой плотности. Для уравнения Больцмана результат Н. Н. Боголюбова не имеет места. Это обстоятельство еще раз подчеркивает необратимый характер уравнения Больцмана и его несовместимость с исходными принципами динамики. Наверное, стоит подчеркнуть, что теорема Боголюбова о сингулярных решениях уравнения Больцмана-Энскога доказана в предположении об отсутствии кратных соударений. Таким образом, это уравнение, действительно, не описывает "полную” динамику системы сталкивающихся шаров. Этот круг вопросов представляет существенный интерес и заслуживает дальнейшего изучения.

В работе [29] доказан аналог теоремы 7.1 для уравнения вихря плоской гидродинамики (3.3)-(3.4). Ищутся решения этого уравнения в виде суммы

$$
\omega(x, y, t)=\sum_{s=1}^{n} \varkappa_{s} \delta\left(x-x_{s}(t)\right) \delta\left(y-y_{s}(t)\right) .
$$

Слагаемое с номером $s$ можно интерпретировать как точечный вихрь интенсивности $\varkappa_{s}$ в точке с координатами $x_{s}, y_{s}$. Обобщенная функция $(7.9)-$ плотность сингулярной меры. Оказывается, если (7.9) удовлетворяет кинетическому уравнению (3.3) с ядром (3.4), то функции $x_{s}(t)$ и $y_{s}(t)$ удовлетворяют 
обыкновенным дифференциальным уравнениям

$$
\varkappa_{s} \dot{x}_{s}=\frac{\partial H}{\partial y_{s}}, \quad \varkappa_{s} \dot{y}_{s}=-\frac{\partial H}{\partial x_{s}} \quad(1 \leqslant s \leqslant n),
$$

где

$$
H=\frac{1}{2 \pi} \sum_{i \neq j} \varkappa_{i} \varkappa_{j} \ln \sqrt{\left(x_{i}-x_{j}\right)^{2}+\left(y_{i}-y_{j}\right)^{2}} .
$$

Это - дифференциальные уравнения Кирхгофа, описывающие динамику $n$ точечных вихрей на плоскости. Они имеют естественную гамильтонову структуру.

Вернемся снова к системе (7.3). Ее фазовое пространство - это прямое произведение $N$ экземпляров многообразия $M$. Положим $Q_{i}(x)=K_{i}(x, x)$.

TEOPEMA 7.2. Если

$$
\sum \frac{\partial Q_{i}}{\partial x_{i}}=0
$$

то фазовый поток системы (7.3) сохраняет "стандартный" фазовый обгем в $M^{N}$ :

$$
d \Lambda=d^{n} x^{(1)} \cdots d^{n} x^{(N)} .
$$

Действительно, с учетом предположения (1.2), дивергенция векторного поля (7.3) на $M^{N}$ равна нулю.

СлЕДСТВИЕ 7.1. Если $K_{i}(x, x)=0$ (отсутствие "самодействия"), то поток (7.3) сохраняет "меру Лиувилля" (7.12).

СлеДСТвИЕ 7.2. Поток системъ (7.3) для кинетического уравнения Гамильтона (1.12) с симметричным ядром сохраняет фазовый обгем.

Действительно, в этом случае условие (7.11), очевидно, выполнено. В частности, следствие 7.2 справедливо и для обычного уравнения Власова.

Теорема 7.2 соответствует гипотезе из $\S 5$ о наличии меры в $L_{2}\left(M, d^{n} x\right)$, инвариантной относительно преобразований $G^{t}, t \in \mathbb{R}$. Следуя схеме рассуждений $\S 6$, мы применим стандартную эргодическую теорию для анализа слабой сходимости по Чезаро семейства сингулярных мер $d \mu_{t}=\rho_{t}(x) d^{n} x$ при $t \rightarrow \infty$, где плотность $\rho_{t}$ задается формулой (7.1).

Итак, рассмотрим функцию времени

$$
\int_{M} \varphi d \mu_{t}
$$

где $\varphi$ - непрерывная функция на $M$. Согласно (7.1), этот интеграл равен

$$
\sum_{j=1}^{N} \varkappa_{j} \varphi\left(x_{1}^{(j)}(t), \ldots, x_{n}^{(j)}(t)\right),
$$

причем $x_{1}^{(j)}, \ldots, x_{n}^{(j)}$ как функции времени удовлетворяют системе дифференциальных уравнений (7.3).

Пусть $M$ замкнуто. Тогда функция $\varphi$ будет заведомо интегрируемой и (согласно индивидуальной эргодической теореме) для почти всех начальных 
данных (начальных расположений сингулярностей) среднее по Чезаро функции (7.13) равно

$$
\sum_{j} \varkappa_{j} \bar{\varphi}_{j}
$$

где

$$
\bar{\varphi}_{j}=\lim _{\tau \rightarrow \infty} \frac{1}{\tau} \int_{0}^{\tau} \varphi\left(x_{1}^{(j)}(t), \ldots, x_{n}^{(j)}(t)\right) d t .
$$

Подчеркнем, что по теореме Крылова-Боголюбова исключительное множество начальных данных не зависит от усредняемой непрерывной функции $\varphi$. Следовательно, для почти всех мер $d \mu_{0}=\rho_{0} d^{n} x$ (которые определяются сингулярностями и коэффициентами $\varkappa_{j}$ ) формула (7.14) задает положительный линейный функционал на пространстве функций, непрерывных на $M$. Применяя теорему Рисса-Радона, получим, что этот функционал есть

$$
\int_{M} \varphi d \bar{\mu}
$$

где $\bar{\mu}$ - некоторая мера на $M$, которая и будет слабым пределом мер $\mu_{t}$ при $t \rightarrow \pm \infty$.

Если мера $\mu_{0}$ вероятностная (т. е. $\sum \varkappa_{j}=1$ ), то предельная мера $\bar{\mu}$ также будет вероятностной. Действительно, достаточно положить $\varphi(x)=1$.

Мера $\bar{\mu}$, конечно, стационарная и инвариантная. Последнее надо понимать в следующем естественном смысле. Пусть

$$
(\bar{\mu})^{N}=\bar{\mu} \times \cdots \times \bar{\mu}
$$

- прямое произведение мер на $M^{N}$ - фазовом пространстве динамической системы (7.3). Тогда ее фазовый поток сохраняет меру $(\bar{\mu})^{N}$.

Эту общую конструкцию, конечно, можно применить к гамильтоновой системе (7.10), описывающей динамику системы точечных вихрей на плоскости. Правда, здесь $M\left(=\mathbb{R}^{2}\right)$ некомпактно. Эту трудность легко преодолеть в рассматриваемом нами случае, когда все интенсивности $\varkappa_{1}, \ldots, \varkappa_{n}$ имеют одинаковый знак: вихри движутся в ограниченной области и никогда не сталкиваются (см., например, [32]).

Динамическую систему (7.10) (как и более общую систему (7.3)) можно рассматривать с точки зрения общей теории ансамблей Гиббса. Более точно это означает следующее. Пусть

$$
\rho_{0}\left(x_{1}, \ldots, x_{n}, y_{1}, \ldots, y_{n}\right)
$$

- начальная плотность распределения вихрей (по Гиббсу) в $2 n$-мерном фазовом пространстве, которая является функцией из $L_{1}$. Пусть $\rho_{t}(x, y)$ - ее значение В текущий момент времени $t$. В теории Гиббса считается, что функция $\rho_{t}$ переносится потоком системы (7.10) или (что то же самое) она удовлетворяет уравнению Лиувилля. Ясно, что $\rho_{t} \in L_{1}$ при всех $t$. Если $\varphi-$ любая ограниченная измеримая функция от переменных $x_{1}, \ldots, x_{n}, y_{1}, \ldots, y_{n}$, то при $t \rightarrow \pm \infty$

$$
\int \ldots \int \rho_{t}(x, y) \varphi(x, y) d^{n} x d^{n} y \rightarrow \int \ldots \int \bar{\rho}(x, y) \varphi(x, y) d^{n} x d^{n} y
$$


причем $\bar{\rho} \in L_{1}$ и

$$
\int \cdots \int \bar{\rho} d^{n} x d^{n} y=\int \cdots \int \rho_{0} d^{n} x d^{n} y=1 .
$$

Подчеркнем, что в (7.15) имеется в виду обычная сходимость по времени, а не более сильная сходимость по Чезаро. Это обстоятельство является следствием однородности динамической системы (7.10): она инвариантна при подстановке

$$
t \mapsto \frac{t}{\lambda}, \quad x_{s} \mapsto \frac{x_{s}}{\lambda}, \quad y_{s} \mapsto \frac{y_{s}}{\lambda} \quad(1 \leqslant s \leqslant n),
$$

где $\lambda$ - произвольный положительный вещественный параметр. При такой подстановке гамильтониан изменяется на некоторую аддитивную константу, а система (7.10) вообще не меняется. Так что система (7.10) будет однородной системой степени однородности -1. Согласно [17], плотность меры, удовлетворяющей уравнению Лиувилля для квазиоднородной системы, имеет слабый предел (в смысле обычной сходимости по времени).

Строго говоря, равенство (7.16) заведомо справедливо, если интенсивности точечных вихрей имеют один знак. Действительно, уравнения Гамильтона (7.10) допускают интеграл момента

$$
I=\sum \varkappa_{s}\left(x_{s}^{2}+y_{s}^{2}\right)
$$

Следовательно, в этом случае инвариантные области

$$
\left\{c_{1} \leqslant I \leqslant c_{2}\right\}
$$

имеют конечную меру и поэтому равенство (7.16) вытекает из теоремы Биркгофа-Хинчина.

В общем случае, когда интенсивности вихрей имеют разные знаки, можно лишь утверждать, что неотрицательный интеграл слева в (7.16) не превосходит единицы. Рассмотрим поучительный пример пары вихрей с интенсивностями $\varkappa$ и $-\varkappa$. Хорошо известно, что в этом случае вихри движутся поступательно с постоянной ненулевой скоростью, которая ортогональна соединяющему их отрезку. Следовательно, $\bar{\rho}=0$.

\section{§ 8. Неравенства для решений кинетического уравнения}

Вернемся к общему кинетическому уравнению (1.1) с условием (1.2). Будем предполагать, что $\rho_{0}(x)>0$ при всех $x \in M$. Из формулы (4.4) вытекает, что тогда $\rho_{t}(x)>0$ при всех $x \in M$ и $t \in \mathbb{R}$.

Пусть $s \mapsto f(s)$ - дважды непрерывно дифференцируемая функция, определенная при всех вещественных $s>0$.

TEOPEMA 8.1. Ecлu $f^{\prime \prime}(s)>0$ nрu $s>0$, mo

$$
\int_{M} f^{\prime}\left(\rho_{0}\right) \rho_{t} d^{n} x \leqslant \int_{M} f^{\prime}\left(\rho_{0}\right) \rho_{0} d^{n} x
$$

для любого решения $\rho_{t}$ уравнения (1.1). Если $f^{\prime \prime}(s)<0$ при $s>0$, то справедливо обратное неравенство. 
Конечно, предполагается, что интеграл слева в (8.1) существует и конечен при всех $t$. Это заведомо выполнено, если $\rho_{0}$ - гладкая функция и фазовое пространство $M$ компактно.

ДОКАЗАТЕЛЬСТво ТЕОРЕмЫ 8.1. Воспользуемся формулой Тейлора с остаточным членом в форме Лагранжа:

$$
f\left(\rho_{t}\right)-f\left(\rho_{0}\right)=f^{\prime}\left(\rho_{0}\right)\left(\rho_{t}-\rho_{0}\right)+\frac{f^{\prime \prime}(\rho)}{2}\left(\rho_{t}-\rho_{0}\right)^{2},
$$

где $\rho>0$. Проинтегрируем обе части этого равенства по фазовому пространству и воспользуемся теоремой 1.1:

$$
\int_{M} f\left(\rho_{t}\right) d^{n} x=\text { const. }
$$

Из (8.2) вытекает теперь, что

$$
\int f^{\prime}\left(\rho_{0}\right) \rho_{t} d^{n} x-\int f^{\prime}\left(\rho_{0}\right) \rho_{0} d^{n} x=\frac{1}{2} \int f^{\prime \prime}(\rho)\left(\rho_{t}-\rho_{0}\right)^{2} d^{n} x .
$$

Если $f^{\prime \prime}>0(<0)$, то правая часть будет неотрицательной (неположительной). Что и требовалось.

СлеДСТВИЕ 8.1. Пусть $\rho_{0} u f^{\prime}\left(\rho_{0}\right)$ - суммируемые с квадратом функиии $u \bar{\rho}$ - слабый предел по Чезаро при $t \rightarrow \pm \infty$ решения $\rho_{t}$ уравнения (1.1). Если $f^{\prime \prime}>0(<0)$, mo

$$
\int_{M} f^{\prime}\left(\rho_{0}\right) \bar{\rho} d^{n} x \leqslant(\geqslant) \int_{M} f^{\prime}\left(\rho_{0}\right) \rho_{0} d^{n} x .
$$

Это - прямое следствие неравенства (8.1), определения слабой сходимости и элементарных свойств суммирования по Чезаро.

Положим теперь $f(s)=s^{2} / 2$. Поскольку $\bar{\rho}$ - стационарное решение уравнения (1.1), то по теореме 1.1

$$
\int \rho_{0} \bar{\rho} d^{n} x=\int \rho_{t} \bar{\rho} d^{n} x
$$

при всех значениях $t$. Переходя еще раз к пределу по Чезаро при $t \rightarrow \pm \infty$, получаем важное

СлЕДСТвиЕ 8.2.

$$
\int_{M} \bar{\rho}^{2} d^{n} x \leqslant \int_{M} \rho_{t}^{2} d^{n} x=\int_{M} \rho_{0}^{2} d^{n} x .
$$

В гидродинамике интеграл

$$
\frac{1}{2} \iint \omega_{t}^{2} d x d y
$$

обычно называют энстрофией; мы будем опускать несущественный множитель $1 / 2$. В случае вязкой жидкости энстрофия пропорциональна скорости 
диссипации энергии. Для идеальной жидкости интеграл (8.5), разумеется, не зависит от времени, а для плоских течений вязкой жидкости он монотонно убывает. Заменяя теперь вихрь $\omega_{t}$ в (8.5) его слабым пределом $\bar{\omega}$, получим значение энстрофии в состоянии предельного статистического равновесия. Согласно (8.4),

$$
\iint \omega_{t}^{2} d x d y \geqslant \iint \bar{\omega}^{2} d x d y
$$

В типичном случае, конечно, будем иметь строгое неравенство.

Неравенство (8.6) выглядит несколько удивительным, поскольку (в отличие от вязкой жидкости) диссипация энстрофии отсутствует. Этот факт имеет существенное значение для понимания развитой двумерной турбулентности. Считается, что некоторая часть "макроскопической” энстрофии переходит в "микроскопическую" энстрофию и что такой механизм диссипации макроскопической инвариантной величины, при котором она передается очень малым флуктуациям, является довольно общим (см., например, [33]). Мы вернемся к этому вопросу в следующем параграфе.

Приведем три примера, раскрывающие общее неравенство (8.1).

А. Пусть $f(s)=s^{p}, p>1$. Тогда неравенство (8.1) принимает вид

$$
\int \rho_{0}^{p-1} \rho_{t} d^{n} x \leqslant \int \rho_{0}^{p} d^{n} x
$$

Оно справедливо для функций $\rho_{0} \in L_{p}$.

Неравенство (8.7) - следствие неравенства Гёльдера:

$$
\int\left(\rho_{0}^{p}\right)^{\frac{1}{q}}\left(\rho_{t}^{p}\right)^{\frac{1}{p}} d^{n} x \leqslant\left[\int \rho_{0}^{p} d^{n} x\right]^{\frac{1}{q}}\left[\int \rho_{t}^{p} d^{n} x\right]^{\frac{1}{p}},
$$

$1 / p+1 / q=1$. Поскольку значения интегралов справа в этом неравенстве совпадают при всех $t$ (теорема 1.1), то из (8.8) вытекает (8.7).

Аналогично доказывается "неравенство Минковского": если $p>1$, то

$$
\int\left(\rho_{0}+\rho_{t}\right)^{p} d^{n} x \leqslant 2^{p} \int \rho_{0}^{p} d^{n} x .
$$

В. Пусть $f(s)=\ln s$ и $M$ компактно. Тогда

$$
\frac{1}{\operatorname{mes} M} \int_{M} \frac{\rho_{t}}{\rho_{0}} d^{n} x \geqslant 1 .
$$

В частности, если функция $\rho_{0}$ непрерывна, то

$$
\frac{1}{\operatorname{mes} M} \int_{M} \frac{\bar{\rho}}{\rho_{0}} d^{n} x \geqslant 1 \text {. }
$$

С. Положим $f(s)=s \ln s, s>0$. Ясно, что $f^{\prime \prime}>0$. Из (8.1) вытекает неравенство

$$
\int_{M} \rho_{t} \ln \rho_{0} d^{n} x \leqslant \int_{M} \rho_{0} \ln \rho_{0} d^{n} x .
$$


Применим это неравенство к решениям обычного кинетического уравнения Власова (1.6). Пусть в начальный момент времени частицы распределены в соответствии с законом Максвелла:

$$
\rho_{0}=c e^{-\frac{v^{2}}{2 k T}}, \quad v^{2}=(v, v),
$$

$c$ - нормировочный множитель. Тогда из (8.9) вытекает любопытное неравенство:

$$
\int \frac{v^{2}}{2} \rho_{t} d x d v \geqslant \int \frac{v^{2}}{2} \rho_{0} d x d v
$$

Оно допускает следующую интерпретацию. Предположим, что в начальный момент времени частицы не взаимодействовали друг с другом (идеальный газ) и находились в состоянии статистического равновесия. Тогда после добавления взаимодействия между частицами это равновесие нарушается и внутренняя энергия системы (пропорциональная ее температуре) может только возрасти. Неравенство (8.10) обобщает неравенство Пуанкаре, установленное им для решений более простого уравнения Лиувилля [34].

\section{§ 9. Грубая энстрофия}

Как уже было сказано, для идеальной жидкости энстрофия (интеграл (8.5), вычисленный по всей плоскости течения) не меняется со временем. С другой стороны, при добавлении сколь угодно малой вязкости энстрофия будет монотонно убывать. Можно ли изучать поведение решений слабо диссипативного двумерного уравнения Навье-Стокса на достаточно больших временах, основываясь на рассмотрении консервативной системы вихрей? Чтобы ответить на этот вопрос положительно, надо как-то “примирить" обратимость уравнений Эйлера и необратимость уравнений Навье-Стокса. Один из путей - переход к "крупнозернистому" описанию завихренности с помощью подходящего локального усреднения поля вихря. Как показывают численные эксперименты, “огрубленная" энстрофия, как правило, убывает со временем (см., например, книги [13], [35] и имеющиеся там ссылки).

Впрочем, в статистической механике эта идея хорошо известна и восходит к Гиббсу. С одной стороны, энтропия Гиббса (1.8) не меняется со временем. С другой стороны, согласно второму началу термодинамики для неравновесных процессов, энтропия замкнутой системы всегда не убывает. Чтобы снять это противоречие, Гиббс предложил заменить исходную плотность распределения вероятностей $\rho_{t}$ грубой плотностью $\hat{\rho}_{t}$, которая получается усреднением $\rho_{t}$ по ячейкам фиксированного разбиения фазового пространства. Плотности $\hat{\rho}_{t}$ можно сопоставить энтропию

$$
\widehat{S}_{t}=-\int \hat{\rho}_{t} \ln \hat{\rho}_{t} d^{n} x,
$$

которую принято называть грубой энтропией. В отличие от тонкой энтроnuи (1.8), она уже может меняться со временем. В двенадцатой главе своей книги [6] Гиббс высказал два свойства грубой энтропии:

1) при измельчении разбиения грубая энтропия стремится к тонкой;

2) грубая энтропия возрастает (точнее, не убывает) с ростом $t$. 
Эти высказывания некритически повторялись многими авторами (см., например, [34]). Однако, как показано в [36], оба эти высказывания в общем случае не верны. Там же указано, как их следует исправить (см. также [3]). Усреднение по ячейкам фиксированного разбиения - не единственный способ огрубления. Более общие способы огрубления плотности вероятностей в классической статистической механике изучались в [37]. Квантовый случай рассматривался в [38]. В плоской гидродинамике идеальной жидкости также используют различные варианты огрубления поля вихря. Так, например, в [35] и [39] рассматривается крупнозернистое усреднение по Гиббсу, а в [24] усреднение проводится по мерам Янга, которые в свое время появились в вариационном исчислении для описания обобщенных экстремалей. Правда, эти работы по статистической гидродинамике выполнены на физическом уровне строгости.

Следуя [36], обсудим теорию грубой энстрофии (по Гиббсу) для общего кинетического уравнения (1.1). Тонкой энстрофией будем называть интеграл

$$
\Omega_{t}=\int_{M} \rho_{t}^{2} d \nu, \quad d \nu=d^{n} x .
$$

Конечно, при этом надо предположить, что $\rho_{0} \in L_{2}(M, d \nu)$. Как мы уже знаем, значение интеграла (9.2) на самом деле не зависит от времени.

Пусть $\left\{M_{j}\right\}, j \in J,-$ разбиение $M$ на измеримые подмножества,

$$
m_{j}=\nu\left(M_{j}\right), \quad 0<\nu\left(M_{j}\right)<\infty, \quad j \in J .
$$

Множество индексов $J$ считается конечным или счетным. Положим

$$
\rho_{j}=\frac{\lambda_{j}}{m_{j}}, \quad \lambda_{j}=\int_{M_{j}} \rho d \nu, \quad \sum \lambda_{j}=1 .
$$

Рассмотрим новую плотность $\hat{\rho}: M \rightarrow \mathbb{R}$ такую, что

$$
\left.\hat{\rho}\right|_{M_{j}}=\rho_{j}, \quad j \in J .
$$

Будем называть $\hat{\rho}$ грубой плотностью. Соответствующая мера $d \hat{\mu}=\hat{\rho} d \nu$ также будет вероятностной:

$$
\int_{M} \hat{\rho} d \nu=\sum \int_{M_{j}} \rho_{j} d \nu=\sum \lambda_{j}=1 .
$$

Определим теперь грубую энстрофию

$$
\widehat{\Omega}=\int_{M} \hat{\rho}^{2} d \nu
$$

Нетрудно проверить, что

$$
\widehat{\Omega}=\sum \frac{\lambda_{j}^{2}}{m_{j}} .
$$

Оказывается, справедливо неравенство

$$
\Omega \geqslant \widehat{\Omega} .
$$


Действительно, по неравенству Коши-Буняковского,

$$
\Omega=\sum_{j} \int_{M_{j}} \rho^{2} d \nu \geqslant \sum_{j} \frac{1}{m_{j}}\left[\int_{M_{j}} \rho d \nu\right]^{2}=\sum m_{j} \rho_{j}^{2}=\widehat{\Omega} .
$$

Неравенство (9.3) ранее уже отмечалось (см., например, [35]). Оно вполне аналогично известному неравенству Гиббса: тонкая энтропия не превосходит грубой энтропии.

Обсудим теперь задачу аппроксимации: когда грубая энстрофия приближает тонкую? Снабдим гладкое конечномерное многообразие $M$ некоторой римановой метрикой и назовем величину

$$
\sup _{j \in J}\left(\operatorname{diam} M_{j}\right) \leqslant \infty
$$

диаметром разбиения $\left\{M_{j}\right\}$ (диаметр вычисляется в метрике пространства $M$ ).

Теорема 9.1. Если многообразие $M$ компактно, то при неограниченном уменьшении диаметра разбиения $\left\{M_{j}\right\}$ грубая энстрофия $\widehat{\Omega}$ с любой наперед заданной точностью аппроксимирует $\Omega$.

Сделаем несколько замечаний.

$1^{\circ}$. Для плоского течения несжимаемой идеальной жидкости аппроксимационная теорема применима в тех случаях, когда область завихренности (где вихрь $\omega$ отличен от нуля) ограничена.

$2^{\circ}$. В общем некомпактном случае теорема 9.1 не справедлива. Однако если плотность $\rho$ достаточно быстро стремится к нулю на бесконечности, грубая энстрофия аппроксимирует тонкую и в некомпактном случае. Точные условия в задаче об аппроксимации энтропии указаны в работе [36].

$3^{\circ}$. В теореме 9.1 условие стремления к нулю диаметра разбиения нельзя заменить условием

$$
\sup _{j \in J}\left(\nu\left(M_{j}\right)\right) \rightarrow 0
$$

Таким образом, теорема 9.1 не является утверждением только из теории меры.

Идея доказательства теоремы состоит в следующем. Пусть $\left\{M_{j}\right\}-$ конечное фиксированное разбиение $M$. Согласно (9.4),

$$
\begin{aligned}
\Omega-\widehat{\Omega} & =\sum_{j}\left[\int_{M_{j}} \rho^{2} d \nu-\frac{1}{m_{j}}\left(\int_{M_{j}} \rho d \nu\right)^{2}\right] \\
& =\frac{1}{2} \sum_{j} \frac{1}{m_{j}} \iint_{M_{j} \times M_{j}}\{\rho(x)-\rho(y)\}^{2} d^{n} x d^{n} y .
\end{aligned}
$$

Известно, что пространство непрерывных функций на компактном многообразии всюду плотно в $L_{2}\left(M, d^{n} x\right)$ (в метрике $L_{2}$ ). Остается воспользоваться свойством равномерной непрерывности функции, непрерывной на компакте, условием

$$
\sup \left(\operatorname{diam} M_{j}\right) \rightarrow 0
$$

и формулой (9.5). 
Перейдем теперь к вопросу о стабилизации грубой плотности при неограниченном возрастании времени. Пусть $\hat{\rho}_{t}$ обозначает грубую плотность (огрубление решения уравнения (1.1)) в текущий момент времени. Будем исходить из следующего предположения:

- для почти всех данных Коши $\rho_{0} \in L_{1}(M, d \nu)$ слабый предел по Чезаро решения уравнения (1.1) существует и также принадлежит $L_{1}(M, d \nu)$.

Это свойство подробно обсуждалось нами в $\S 6$. Чтобы упростить дальнейшие обозначения, слабый предел $\bar{\rho}$ обозначим $\rho_{\infty}$; пусть $\hat{\rho}_{\infty}-$ огрубление этой функции по Гиббсу относительно фиксированного разбиения $\left\{M_{j}\right\}$.

Теорема 9.2. Для почти всех решений уравнения (1.1)

$$
\lim _{\tau \rightarrow \pm \infty} \frac{1}{\tau} \int_{0}^{\tau} \hat{\rho}_{t} d t=\hat{\rho}_{\infty} .
$$

Действительно, для всех $x \in M_{j}$

$$
\hat{\rho}_{t}=\int_{M} \rho_{t} \varphi_{j} d \nu
$$

где $\varphi_{j}$ - характеристическая функция измеримого множества $M_{j}$. Для доказательства формулы (9.6) остается воспользоваться определением слабой сходимости (по Чезаро).

Обсудим, наконец, задачу об убывании грубой энстрофии

$$
\widehat{\Omega}_{t}=\int_{M} \hat{\rho}_{t}^{2} d \nu
$$

при возрастании времени. В отличие от тонкой энстрофии (9.2), интеграл (9.7) уже, как правило, зависит от времени. Положим

$$
\widehat{\Omega}_{\infty}=\int_{M} \hat{\rho}_{\infty}^{2} d \nu .
$$

Согласно (8.4),

$$
\Omega_{\infty} \leqslant \Omega_{t}=\Omega_{0}
$$

ТеОрема 9.3. Пусть $M$ компактно и $\rho_{t}$ - решение обобщенного кинетического уравнения (1.1) такое, что

1) $\rho_{0} \in L_{2}(M, d \nu)$,

2) $\rho_{t}$ имеет слабый предел по Чезаро $\rho_{\infty} \in L_{2}(M, d \nu)$.

Если неравенство (9.8) строгое, то при достаточно мальх $\sup \left(\operatorname{diam} M_{j}\right)$ справедливо неравенство

$$
\widehat{\Omega}_{\infty}<\widehat{\Omega}_{0}
$$

Это утверждение просто выводится из аппроксимационной теоремы 9.1. Повидимому, квадратичная суммируемость слабого предела $\rho_{\infty}$ имеет место для почти всех решений уравнения (1.1) (если, конечно, $\left.\rho_{0} \in L_{2}\right)$.

В заключение отметим, что грубая энстрофия $\widehat{\Omega}_{t}$ убывает, конечно, не монотонно. Например, пусть (1.1) - это уравнение Лиувилля для нелинейной 
гамильтоновой системы с квазиоднородным гамильтонианом. Тогда, как доказано в [17], пределы

$$
\lim _{t \rightarrow+\infty} \widehat{\Omega}_{t} \quad \text { и } \quad \lim _{t \rightarrow-\infty} \widehat{\Omega}_{t}
$$

существуют и совпадают. Следовательно, если функция $t \mapsto \widehat{\Omega}_{t}$ убывает на каком-то интервале времени, то обязательно найдется интервал, где эта функция возрастает.

\section{§ 10. Тождество Лагранжа}

Обратимся вновь к кинетическому уравнению Власова (1.6), которое описывает эволюцию континуума взаимодействующих частиц. Как уже отмечалось в 7 , это уравнение допускает точное обобщенное решение в виде конечной взвешенной суммы $\delta$-функций Дирака, которое соответствует динамике Ньютона конечного числа частиц.

Итак, пусть вначале система состоит из $n$ взаимодействующих частиц с массами $m_{1}, \ldots, m_{n}$ (интенсивности сингулярной меры вида (7.1)) и радиус-векторами $r_{1}, \ldots, r_{n}$ (сингулярности этой меры) относительно некоторой неподвижной точки евклидова пространства $E$ (его размерность для дальнейшего не имеет значения). Предполагается, что силы потенциальны и потенциальная энергия $V$ - однородная функция от $r_{1}, \ldots, r_{n}$ степени однородности $m$. Знаменитое тождество Лагранжа имеет вид

$$
\ddot{I}=4 T-2 m V
$$

где

$$
I=\sum m_{i}\left(r_{i}, r_{i}\right)=\sum m_{i} r_{i}^{2}
$$

- момент инерции системы точек относительно начала системы отсчета, а

$$
T=\frac{1}{2} \sum m_{i}\left(\dot{r}_{i}, \dot{r}_{i}\right)
$$

- ее кинетическая энергия.

Как показано в [40; гл. 3], соотношение (10.1) тесно связано с действием группы гомотетий

$$
r_{i} \mapsto \alpha r_{i} \quad(1 \leqslant i \leqslant n), \quad \alpha>0 .
$$

При такой замене $T \mapsto \alpha^{2} T$ и $V \mapsto \alpha^{m} V$. Хотя лагранжиан $T+V$ не допускает группу гомотетий, тождество Лагранжа легко выводится из некоторого естественного обобщения теоремы Нётер.

С учетом интеграла энергии

$$
T+V=h
$$

равенство (10.1) представляется в виде

$$
\ddot{I}=4 h-2(m+2) V .
$$

Из тождества Лагранжа вытекает ряд важных следствий. Например, для гравитационного взаимодействия $m=-1$ и $V<0$. Следовательно, если $h \geqslant 0$, то, 
согласно (10.3), $\ddot{I}>0$. Отсюда вытекает известный результат Якоби о неустойчивости системы гравитирующих тел с неотрицательным запасом полной энергии: некоторые частицы либо неограниченно сближаются, либо уходят друг от друга на бесконечное расстояние.

Другое важное следствие справедливо в предположении компактности энергетической поверхности (10.2) в фазовом пространстве рассматриваемой системы. В этом случае $V$ и $T$ как функции времени сходятся по Чезаро при $t \rightarrow \pm \infty$ соответственно к

$$
\frac{2 h}{m+2} \quad \text { и } \quad \frac{m h}{m+2} .
$$

Этот результат, полученный еще Клаузиусом, примечателен тем, что для функций $T$ и $V$ средние по времени совпадают со средним по пространству (по компактному энергетическому многообразию) независимо от свойства эргодичности рассматриваемой системы. Статистический вариант теоремы Клаузиуса, основанный на рассмотрении ансамблей Гиббса, указан в работе [41].

Следуя [42], покажем, как (с некоторыми предосторожностями) тождество Лагранжа можно распространить на континуум взаимодействующих частиц. Вместо дискретного набора масс вводим непрерывное распределение с плотностью $\rho(x, v, t)$, где $x$ - точка евклидова пространства, $v$ - ее скорость. Будем считать выполненным условие нормировки

$$
\int \rho(x, v, t) d x d v=1 \text {. }
$$

Поскольку $\rho$ удовлетворяет уравнению Власова, то интеграл слева в (10.4) не зависит от времени. Впрочем, это очевидно с физической точки зрения, поскольку частицы не исчезают и не рождаются.

Пусть сила $F$ в уравнении (1.6) имеет вид

$$
F=-\frac{\partial}{\partial x} \int W(|x-y|) \rho(y, u, t) d y d u .
$$

Здесь $W$ - плотность потенциала парного взаимодействия.

Введем момент инерции континуальной системы частиц относительно начала системы отсчета

$$
I(t)=\int \rho(x, v, t) x^{2} d x d v
$$

а также кинетическую и потенциальную энергию согласно формулам (1.10а) и $(1.10 \mathrm{~b})$, где симметричное ядро $K(x, y)$ равно $W(|x-y|)$. Согласно (1.11), полная энергия системы $T(t)+V(t)$ не меняется со временем. Будем предполагать, что $\rho$ - гладкая функция от своих переменных, интегралы, задающие $I, T$ и $V$, сходятся и гладко зависят от времени.

Теорема 10.1. Если $W$ - однородная функиия степени $m$, то справедливо тождество Лагранжа (10.1). 
ДокАЗАТЕЛьство. Используя уравнение Власова (1.6), вычислим сначала производную по времени от (10.5):

$$
\begin{aligned}
\dot{I}=\int & \frac{\partial \rho}{\partial t} x^{2} d x d v=-\int\left(\frac{\partial \rho}{\partial x}, v\right) x^{2} d x d v \\
& +\int x^{2}\left(\frac{\partial \rho}{\partial v}, \frac{\partial}{\partial x} \int W(|x-y|) \rho(y, u, t) d y d u\right) d x d v
\end{aligned}
$$

По формуле Гаусса-Остроградского первый интеграл равен

$$
2 \int(x, v) \rho(x, v, t) d x d v
$$

а второй равен нулю (достаточно провести интегрирование по $v$ ).

Аналогично, вторая производная интеграла (10.5) равна

$$
\begin{aligned}
& -2 \int\left(\frac{\partial \rho}{\partial x}, v\right)(x, v) d x d v \\
& \quad+2 \int(x, v)\left(\frac{\partial \rho}{\partial v}, \frac{\partial}{\partial x} \int W(|x-y|) \rho(y, u, t) d y d u\right) d x d v
\end{aligned}
$$

Снова применяя формулу Гаусса-Остроградского, первый интеграл преобразуем к виду

$$
2 \int(v, v) \rho d x d v=4 T .
$$

Второй станет равным

$$
2 \int \rho(x, v, t)\left(x, \frac{\partial}{\partial x} \int W(|x-y|) \rho(y, u, t) d y d u\right) d x d v
$$

Поскольку $W$ - однородная функция от

$$
|x-y|=\left(\sum\left(x_{i}-y_{i}\right)^{2}\right)^{\frac{1}{2}}
$$

Tо

$$
\left(x, \frac{\partial W}{\partial x}\right)+\left(y, \frac{\partial W}{\partial y}\right)=m W .
$$

Далее, значение интеграла (10.7) не изменится, если в подынтегральной функции поменять местами две группы переменных: $x, v$ и $y, u$. Применяя затем формулу (10.8), интеграл (10.7) сведем к

$$
m \int \rho(x, v, t) \rho(y, u, t) W(|x-y|) d x d v d y d u=2 m V
$$

Что и доказывает теорему.

ЗАмЕчАниЕ. При доказательстве теоремы 10.1 существенную роль играли предположение о гладкости решения уравнения Власова и формула ГауссаОстроградского. Было бы интересным доказать тождество Лагранжа для обобщенных решений кинетического уравнения. Для этого нужна другая техника. 
В предположении ограниченности производной $\dot{I}$ и интеграла энергии $T+$ $V=h$ (теорема 1.2) из теоремы 10.1 выводится упомянутая выше теорема Клаузиуса. Правда, в отличие от конечномерного случая, это условие в общем случае труднопроверяемое. Укажем некоторые достаточные условия ограниченности $\dot{I}$.

Если сам момент инерции $I$ ограничен и потенциальная энергия неотрицательна (например, $W \geqslant 0$ ), то функция $|\dot{I}(t)|$ ограничена на всей оси времени. Действительно, по неравенству Коши $2|(x, v)| \leqslant(x, x)+(v, v)$, из (10.6) вытекает оценка

$$
|\dot{I}| \leqslant I+2 T \text {. }
$$

Остается заметить, что из формулы (1.11) с учетом неравенства $V \geqslant 0$ вытекает ограниченность кинетической энергии $(T \leqslant h)$.

В качестве простого примера рассмотрим континуум частиц в евклидовом пространстве $E=\{x\}$, упруго притягивающихся друг к другу. Эта задача рассматривалась в $\S 2$. Плотность потенциала $W$ равна

$$
\frac{k}{2}|x-y|^{2}=\frac{k}{2} \sum\left(x_{i}-y_{i}\right)^{2},
$$

где $k=$ const $>0$ - коэффициент упругости; $W$ - однородная функция степени $m=2$.

С учетом соглашения о нормировке (10.4) центр масс системы определяется вектором

$$
\xi(t)=\int x \rho(x, v, t) d x d v
$$

Как показано в $\S 2, \ddot{\xi}=0$. Без ущерба для общности будем считать, что $\xi=0$. Это предположение эквивалентно переходу в инерциальную систему отсчета, движущуюся вместе с центром масс. С учетом этого соглашения и формулы (10.4) для упругого взаимодействия суммарная потенциальная энергия (1.10b) равна

$$
\begin{gathered}
\frac{k}{4} \int \rho(x, v, t) \rho(y, u, t) x^{2} d x d v d y d u+\frac{k}{4} \int \rho(x, v, t) \rho(y, u, t) y^{2} d x d v d y d u \\
-\frac{k}{2} \sum \int x_{i} \rho(x, v, t) d x d v \int y_{i} \rho(y, u, t) d y d u=\frac{k}{2} I .
\end{gathered}
$$

Тогда по теореме 10.1 и формуле (10.3)

$$
\ddot{I}=4 h-4 k I \text {. }
$$

Следовательно, момент инерции континуума осцилляторов относительно его центра масс совершает гармонические колебания с частотой $2 \sqrt{k}$, а его среднее значение равно $h / k$. Поскольку момент инерции ограничен, то полная энергия в среднем распределяется поровну между ее кинетической и потенциальной частями:

$$
\lim _{\tau \rightarrow \infty} \frac{1}{\tau} \int_{0}^{\tau} T(t) d t=\lim _{\tau \rightarrow \infty} \frac{1}{\tau} \int_{0}^{\tau} V(t) d t=\frac{h}{2} .
$$


В заключение сделаем следующее замечание. Если плотность потенциала $W$ - однородная функция степени $m$, то отсюда сразу не видно, почему потенциальная энергия (1.10b) также будет однородным функционалом. Дело в том, что в выражение для $V$ входит плотность распределения, также зависящая от положения частиц. Выход из этого затруднения состоит в следующем наблюдении: кинетическое уравнение Власова (1.6) допускает группу подобий

$$
t \mapsto \alpha^{\frac{2-m}{2}} t, \quad x \mapsto \alpha x, \quad v \mapsto \alpha^{\frac{m}{2}} v, \quad \rho \mapsto \alpha^{-\frac{m+2}{2} n} \rho,
$$

где $\alpha$ - произвольный положительный параметр, $n=\operatorname{dim} E$. Более того, при таких преобразованиях не меняется также и нормировочное соотношение (10.4), которое выражает закон сохранения общей массы частиц.

В результате преобразования (10.9) интегральные величины $I, T$ и $V$ умножаются на $\alpha^{2}, \alpha^{m}$ и $\alpha^{m}$ соответственно. В частности, суммарная потенциальная энергия континуума частиц будет однородным функционалом нужной степени $m$.

\section{Список литературы}

[1] Р. Л. Добрушин, Я.Г. Синай, Ю.М.Сухов, “Динамические системы статистической механики”, Динамические системы-2, Итоги науки и техники. Соврем. проблемы матем. Фундам. напр., 2, ВИНИТИ, М., 1985, 233-284; англ. пер.: R. L. Dobrushin, Ya.G. Sinai, Yu. M. Sukhov, "Dynamical systems of statistical mechanics", Dynamical systems. II. Ergodic theory with applications to dynamical systems and statistical mechanics, Encyclopaedia Math. Sci., 2, Springer-Verlag, Berlin, 1989, 207-278.

[2] В.В. Козлов, Тепловое равновесие по Гиббсу и Пуанкаре, НИЦ "Регулярная и хаотическая динамика", Ижевск, 2002.

[3] В. В. Козлов, Ансамбли Гиббса и неравновесная статистическая механика, НИЦ "Регулярная и хаотическая динамика", М.-Ижевск, 2008.

[4] V. V. Kozlov, D. V. Treschev, "On new forms of the ergodic theorem", J. Dynam. Control Systems, 9:3 (2003), 449-453.

[5] В. И. Богачев, А. В. Королев, "Об эргодической теореме в форме Козлова-Трещева", Докл. РАН, 412:3 (2007), 295-301.

[6] Дж. В. Гиббс, Основные принципы статистической механики, излагаемые со специальным применением к рациональному обоснованию термодинамики, НИЦ "Регулярная и хаотическая динамика", М., 2002; пер. с англ.: J.W. Gibbs, Elementary principles in statistical mechanics developed with especial reference to the rational foundation of thermodynamics, Charles Scribners Sons, New York, 1902.

[7] Дж. Уленбек, Дж. Форд, Лекиии по статистической механике, Мир, М., 1965; пер. с англ.: G. E. Uhlenbeck, G. W. Ford, Lectures in statistical mechanics, Proceedings of the Summer Seminar (Boulder, Colorado, 1960), Lectures in Appl. Math., 1, Amer. Math. Soc., Providence, RI, 1963.

[8] А.А. Власов, Статистические функиии распределения, Наука, М., 1966.

[9] В. В. Веденяпин, Кинетические уравнения Больимана и Власова, Физматлит, М., 2001.

[10] В. П. Маслов, "Уравнение самосогласованного поля”, Итоги науки и техники. Соврем. проблемы матем., 11, ВИНИТИ, М., 1978, 153-234; англ. пер.: V. P. Maslov, "Equations of the self-consistent field", J. Sov. Math., 11 (1979), 123-195.

[11] E. Hölder, "Über die unbeschränkte Fortsetzbarkeit einer stetigen ebenen Bewegung in einer unbegrenzten inkompressiblen Flüssigkeit", Math. Z., 37:1 (1933), 727-738. 
[12] W. Wolibner, "Un théorème sur l'existence du mouvement plan d'un fluide parfait, homogène, incompressible, pendant un temps infiniment long", Math. Z., 37:1 (1933), 698-726.

[13] У. Фриш, Турбулентность. Наследие А.Н. Колмогорова, ФАЗИС, М., 1998; пер. с англ.: U. Frisch, Turbulence. The legacy of A. N. Kolmogorov, Cambridge, Cambridge Univ. Press, 1995.

[14] В. И. Юдович, "О проблемах и перспективах современной математической гидродинамики", Успехи механики, 1 (2002), 61-102.

[15] W. Braun, K. Hepp, "The Vlasov dynamics and its fluctuations in the $1 / N$ limit of interacting classical particles", Comm. Math. Phys., 56:2 (1977), 101-113.

[16] Р. Л. Добрушин, “Уравнения Власова”, Функи. анализ и его прил., 13:2 (1979), 48-58; англ. пер.: R. L. Dobrushin, "Vlasov equations", Funct. Anal. Appl., 13:2 (1979), 115-123.

[17] В. В. Козлов, Д. В. Трещёв, “Слабая сходимость решений уравнений Лиувилля для нелинейных гамильтоновых систем”, ТМФ, 134:3 (2003), 388-400; англ. пер.: V.V. Kozlov, D. V. Treshchev, "Weak convergence of solutions of the Liouville equation for nonlinear Hamiltonian systems", Theoret. and Math. Phys., 134:3 (2003), 339-350.

[18] В.И. Богачев, Гауссовские меры, Наука, Физматлит, М., 1997; англ. пер.: V.I. Bogachev, Gaussian measures, Math. Surveys Monogr., 62, Amer. Math. Soc., Providence, RI, 1998.

[19] А. М. Вершик, “Существует ли мера Лебега в бесконечномерном пространстве?", Тр. МИАН, 259 (2007), 256-281; англ. пер.: A. M. Vershik, "Does there exist a Lebesgue measure in the infinite-dimensional space?", Proc. Steklov Inst. Math., 259:2 (2007), 248-272.

[20] В. В. Немыцкий, В.В. Степанов, Качественная теория дифференииальных уравнений, Гостехиздат, М.-Л., 1947.

[21] A. Ionescu Tulcea, "Ergodic properties of isometries in $L_{p}$-spaces, $1<p<\infty$ ", Bull. Amer. Math. Soc., 70 (1964), 366-371.

[22] M. A. Akcoglu, "A pointwise ergodic theorem in $L_{p}$-spaces", Canad. J. Math., 27:5 (1975), 1075-1082.

[23] D. Montgomery, G. Joyce, "Statistical mechanics of 'negative temperature' states", Phys. Fluids, 17:6 (1974), 1139-1145.

[24] R. Robert, J. Sommeria, "Statistical equilibrium states for two-dimensional flows", J. Fluid Mech., 229 (1991), 291-310.

[25] M. B. Isichenko, "Percolation, statistical topography, and transport in random media", Rev. Modern Phys., 64:4 (1992), 961-1043.

[26] G. L. Eyink, H. Spohn, "Negative temperature states and large-scale long-lived vortices in two-dimensional turbulence", J. Statist. Phys., 70:3-4 (1993), 833-886.

[27] Л. Онзагер, "Статистическая гидродинамика", Проблемъ турбулентности. Сборник переводных работ, НИЦ "Регулярная и хаотическая динамика", M.-Ижевск, 2006, 196-207; пер. с англ.: L. Onsager, "Statistical hydrodynamics", Nuovo Cimento (9), 6:2 (1949), 279-287.

[28] H. Marmanis, "The kinetic theory of point vortices", Proc. R. Soc. Lond. Ser. A Math. Phys. Eng. Sci., 454 (1998), 587-606.

[29] V. V. Kozlov, "Vorticity equation of 2D-hydrodynamics, Vlasov steady-state kinetic equation and developed turbulence", IUTAM Symposium on Hamiltonian Dynamics, Vortex Structures, Turbulence (Moscow, August 25-30, 2006), Springer, 2008, 27-37.

[30] А. А. Власов, Теория многих частии, Гостехиздат, М., 1950. 
[31] Н. Н. Боголюбов, "Микроскопические решения уравнения Больцмана-Энскога в кинетической теории для упругих шаров", ТМФ, 24:2 (1975), 242-247; англ. пер.: N. N. Bogolyubov, "Microscopic solutions of the Boltzmann-Enskog equation in kinetic theory for elastic balls", Theoret. and Math. Phys., 24:2 (1975), 804-807.

[32] Е.А. Новиков, “Динамика и статистика системы вихрей”, ЖЭТФ, 68:5 (1975), 1868-1882.

[33] Y. Pomeau, "Asymptotic time behaviour of nonlinear classical field equations", Nonlinearity, 5:3 (1992), 707-720.

[34] А. Пуанкаре, "Замечания о кинетической теории газов”, Избранные труды. Т. 3: Математика. Теоретическая физика. Анализ математических и естественнонаучных работ Анри Пуанкаре, Классики науки, Наука, М., 1974, 385-412; пер. с франц.: H. Poincaré, "Réflexions sur la théorie cinétique des gaz", J. de Phys., Sér. 4, 5 (1906), 369-403.

[35] C. C. Lim, J. Nebus, Vorticity, statistical mechanics, and Monte Carlo simulations, Springer Monogr. Math., Springer, New York, 2007.

[36] В. В. Козлов, Д. В. Трещев, “Тонкая и грубая энтропия в задачах статистической механики", ТМФ, 151:1 (2007), 120-137; англ. пер.: V. V. Kozlov, D. V. Treshchev, "Fine-grained and coarse-grained entropy in problems of statistical mechanics", Theoret. and Math. Phys., 151:1 (2007), 539-555.

[37] G. N. Piftankin, D. V. Treschev, "Gibbs entropy and dynamics", Chaos, 18:2 (2008), 023116, $10 \mathrm{p}$.

[38] В. В. Козлов, О. Г. Смолянов, "Слабая сходимость состояний в квантовой статистической механике", Докл. РАН, 417:2 (2007), 180-184; англ. пер.: V. V. Kozlov, O. G. Smolyanov, "Weak convergence of states in statistical quantum mechanics", Dokl. Math., 76:3 (2007), 958-961.

[39] J. Miller, "Statistical mechanics of Euler equations in two dimensions", Phys. Rev. Lett., 65:17 (1990), 2137-2140.

[40] В.И. Арнольд, В. В. Козлов, А. И. Нейштадт, Математические аспекты классической и небесной механики, Эдиториал УРСС, М., 2002; англ. пер.: V.I. Arnold, V.V. Kozlov, A.I. Neishtadt, Mathematical aspects of classical and celestial mechanics, Encyclopaedia Math. Sci., 3, Springer, Berlin, 2006.

[41] В. В. Козлов, "Ансамбли Гиббса, равнораспределенность энергии симпатических осцилляторов и статистические модели термостата", Нелинейная динамика, 3:2 (2007), 123-140.

[42] В.В. Козлов, "Тождество Лагранжа и его обобщения", Нелинейная динамика, 4:2 (2008), 157-168; англ. пер.: V.V. Kozlov, "Lagrange's identity and its generalizations", Regul. Chaotic Dyn., 13:2 (2008), 71-80.

В. В. Козлов (V. V. Kozlov)

Математический институт им. В. А. Стеклова РАН

Поступила в редакцию

E-mail: kozlov@pran.ru

21.05.2008 